\title{
Stability and Sensitivity Analysis of a Plant Disease Model with Continuous Cultural Control Strategy
}

\author{
Zhang Zhonghua and Suo Yaohong \\ School of Sciences, Xian University of Science and Technology, Xian 710054, China \\ Correspondence should be addressed to Zhang Zhonghua; wwwzhangzhonghua@163.com
}

Received 12 November 2013; Revised 15 January 2014; Accepted 16 January 2014; Published 16 March 2014

Academic Editor: Zhijun Liu

Copyright (C) 2014 Z. Zhonghua and S. Yaohong. This is an open access article distributed under the Creative Commons Attribution License, which permits unrestricted use, distribution, and reproduction in any medium, provided the original work is properly cited.

In this paper, a plant disease model with continuous cultural control strategy and time delay is formulated. Then, how the time delay affects the overall disease progression and, mathematically, how the delay affects the dynamics of the model are investigated. By analyzing the transendental characteristic equation, stability conditions related to the time delay are derived for the diseasefree equilibrium. Specially, when $R_{0}=1$, the Jacobi matrix of the model at the disease-free equilibrium always has a simple zero eigenvalue for all $\tau \geq 0$. The center manifold reduction and the normal form theory are used to discuss the stability and the steadystate bifurcations of the model near the nonhyperbolic disease-free equilibrium. Then, the sensitivity analysis of the threshold parameter $R_{0}$ and the positive equilibrium $E^{*}$ is carried out in order to determine the relative importance of different factors responsible for disease transmission. Finally, numerical simulations are employed to support the qualitative results.

\section{Introduction}

Viral disease is a key constraint on the production of staple food crop in the lesser developed countries. Diseases caused by plant viruses in cassava (Manihot esculenta), sweet potato (Ipomoea batatas) and plantain (musa spp.) are among the main constraints on sustainable production of these vegetatively propagated staple food crops, see Rybicki and Pietersen [1], Dahal et al. [2], Gibson and Aritua [3], and Thresh and Cooter [4]. Furthermore, new viral strains frequently emerge, some of which bring devastating consequences, such as the current pandemic of the virus causing cassava mosaic disease (CMD) in Africa, see Gibson et al. [5]. Governments and farmers have been evolving practices for combatting with the plagues suffered by crops. The growing understanding of the interactions between pathogen and host has enabled us to develop a wide array of measures for the control of specific plant diseases. Large investments are underway to alleviate poverty and malnutrition by developing new or more effective control strategies, which include plant breeding for resistance to the virus, control of vectors as well as crop sanitation through removal of diseased plants, and improved selection of planting material for these vegetatively propagated crops. Such experiences have led to the development of integrated management concepts for virus diseases that combine available host resistance, cultural, chemical, and biological control measures. Examples of how epidemiological information can be used to develop effective integrated disease management (IDM) strategies for diverse situations have been described in [6-9]. IDM involves the selection and application of a wide range of control strategies that minimize losses and maximize returns. A cultural control strategy including replanting or roguing diseased plants is a widely accepted treatment for plant epidemics which involves periodic inspections followed by removal of the infected plants.

Mathematical models of plant-virus epidemics were developed to provide detailed explanation on how to describe, analyze, and predict epidemics of plant disease for the ultimate purposes of developing and testing control strategies and tactics for crop protection, see van den Bosch et al. [10], Chan and Jeger [11], Fishman et al. [12], and the references cited therein. A simple model for plant disease with 
a continuous cultural control strategy, such as replanting and roguing or removing, is as follows:

$$
\begin{gathered}
\frac{\mathrm{d} S(t)}{\mathrm{d} t}=\sigma \phi-\beta S(t) I(t)-\eta S(t), \\
\frac{\mathrm{d} S(t)}{\mathrm{d} t}=\sigma(1-\phi)+\beta S(t) I(t)-(\eta+\omega) I(t),
\end{gathered}
$$

where $S$ and $I$ denote the respective number of the susceptible and infected plants and $\beta$ is the transmission rate. Crop is planted from in vitro propagated virus free material, from cuttings taken from a previous crop, or from a combination of these methods. New plants enter the system by continual replanting at rate $\sigma$ with a proportion $\phi$ for the susceptible plants and $1-\phi$ for the infected ones. Removal occurs for sanitation at rate $\omega$ or for death at rate $\eta$, and $1 / \eta$ denotes either harvest time or the end of reproductive lifetime of plants. Neglecting the biological meaning of the parameters, (1) can be seen as an SI human disease model with immigration of the infective individuals and its extended forms have been extensively studied, for example, van den Bosch et al. [10], Brauer and van den Driessche [13], Tang et al. [14], and the references cited therein.

In [12], Fishman et al. divided the time axis $(0, \infty)$ into equal periods of length $T$ and developed a model with periodic control strategy as follows:

$$
\frac{\mathrm{d} x_{i}(t)}{\mathrm{d} t}=A x_{i}(t)\left(1-x_{i}(t)\right), \quad 0 \leq t \leq T,
$$

where $A$ is the infection rate and $x_{i}(t)$ is the ratio between the number $n_{i}(t)$ of the infected trees at instant $t$ of period $i$ and the total number $N_{i}$ of trees at the beginning of the period. Equation (2) characterizes the temporal spread of an epidemic in a closed plant population with periodic removal of infected plants and has an application to the spread of citrus tristeza virus disease. Economic evaluations and comparisons between two policies, eradication or no eradication, were given, and further simulations of the model and sensitivity analysis for a wide range of parameters were presented. They concluded that the discovery-eradication program is economically justified and superior to allowing the disease to progress unchecked. The results were helpful in evaluating policies of controlling the disease, and (2) could be modified to simulate other plant epidemics with periodic treatments.

Based on [12], Tang et al. in [14] proposed periodic pulse replanting and roguing strategies and changed (1) into the following:

$$
\begin{gathered}
\frac{\mathrm{d} S(t)}{\mathrm{d} t}=-\beta S(t) I(t)-\eta S(t), \quad t \neq n T, \\
\frac{\mathrm{d} I(t)}{\mathrm{d} t}=\beta S(t) I(t)-\eta I(t), \quad t \neq n T, \\
S\left(n T^{+}\right)=S(n T)+\sigma \phi, \quad t=n T, \\
I\left(n T^{+}\right)=(1-\omega) I(n T)+\sigma(1-\phi) \phi, \quad t=n T,
\end{gathered}
$$

where $n=1,2, \ldots$, and $T$ is a fixed positive constant and denotes the period of the impulsive effect. The sufficient conditions under which the infected plant free periodic solution with fixed moments is globally stable were obtained.

It can be seen that the reversion of the infected plants is not considered in the above models. In [10], to study the effect of the reversion of infected plants on the transmission of disease, van den Bosch et al. constructed the following model:

$$
\begin{aligned}
\frac{\mathrm{d} S(t)}{\mathrm{d} t}= & \sigma \phi+\sigma(1-\phi) \frac{r(1-p) I(t)+S(t)}{(1-p) I(t)+S(t)} \\
& -\beta S(t) I(t)-\eta S(t), \\
\frac{\mathrm{d} I(t)}{\mathrm{d} t}= & \sigma(1-\phi) \frac{(1-r)(1-p) I(t)}{(1-p) I(t)+S(t)}+\beta S(t) I(t) \\
& -(\eta+\omega) I(t) .
\end{aligned}
$$

Some cuttings from infected plants may be healthy due to reversion with probability $r$. Cuttings are selected visually or using diagnostic methods and discarded with probability $p$. They found a threshold parameter $R_{0}$ and proved that (4) owns a unique positive equilibrium when $R_{0}>1$, and its stability was numerically checked. They have shown that the development of new and improved disease control methods for viral diseases of vegetatively propagated staple food crops ought to take evolutionary responses of the virus into consideration. Not doing so leads to a risk of failure, which can result in considerable economic losses and increased poverty. Specifically in vitro propagation, diagnostics, and breeding methods carry a risk of failure due to the selection for virus strains that build up a high within-plant virus titre. For vegetatively propagated crops, sanitation by roguing has a low risk of failure owing to its combination of selecting for low virus titre strains as well as increasing healthy crop density.

Latent infection of plants by pathogens has been recognized for many years and it is often considered one of the highest levels of parasitism, since the host and the parasite coexist with minimal damage to the host, see Sinclair [15]. Latent infection is important in the epidemiology, the control of the plant diseases, and also in breeding for resistance or tolerance to a pathogen; see Chan and Jeger [11]. An understanding of latent infection contributes to development of effective control measures, as does an understanding of penetration, colonization, disease expression, and yield losses. Many plant diseases, such as chlorotic leaf distortion of sweet potato, citrus black spot, Colletotrichum gloeosporioides, Alternaria alternata, possess latent period, that is, the time elapsed between exposure to a pathogenic organism and when symptoms and signs are first apparent; see Ames et al. [16], Miles et al. [17], Chakraborty [18], and Karaoglanidis et al. [19]. The earliest plant disease model is due to van der Plank [20], and in which he used a delay differential equation to represent $I(t)$, the density of host tissue first infected at or before time $t$,

$$
\frac{\mathrm{d} I(t)}{\mathrm{d} t}=R(I(t-p)-I(t-p-i))(1-I(t)) .
$$

The latent period $p$ and the infectious period $i$ are constant, and the parameter $R$ is the corrected basic rate of infection. 
The key threshold is the progeny-parent ratio, $i R$ : a value $>$ 1 indicates that the density of infected tissue will increase. Unfortunately delay differential equations are difficult to analyze; see Madden [21] and Murray [22] and, despite widespread adoption of discrete time approximations to van der Plank's model in early simulations of plant disease, see Teng [23], Zadoks [24] and a number of often subtle mathematical analyses that followed by Jeger $[25,26]$, Kushalappa and Ludwig [27], Waggoner [28], the model is now rarely used in theoretical studies. However, it is so influential and still of significant historical interest; see Cunniffe et al. [29].

To our knowledge, how the latent period affects the dynamics of (4) remains unknown. Motivated by this, using a time delay $\tau$ to denote the latent period of plant disease and replacing the incidence rate of the disease by $\beta S(t-\tau) I(t-\tau)$ we formulate the following model:

$$
\begin{aligned}
\frac{\mathrm{d} S(t)}{\mathrm{d} t}= & \sigma \phi+\sigma(1-\phi) \frac{r(1-p) I(t)+S(t)}{(1-p) I(t)+S(t)} \\
& -\beta S(t-\tau) I(t-\tau)-\eta S(t), \\
\frac{\mathrm{d} I(t)}{\mathrm{d} t}= & \sigma(1-\phi) \frac{(1-r)(1-p) I(t)}{(1-p) I(t)+S(t)}+\beta S(t-\tau) I(t-\tau) \\
& -(\eta+\omega) I(t) .
\end{aligned}
$$

The remainder of the paper is arranged as follows. Section 2 discusses the stability of the disease-free equilibrium under the conditions of $R_{0} \neq 1$, or $R_{0}=1, \tau=0$; Section 3 computes the normal forms on the center manifold and investigates the dynamical behaviors of system (6) near the disease-free equilibrium when $R_{0}=1, \tau>0$; Section 4 is the sensitivity analysis of $R_{0}$ and the positive equilibrium to all of the parameters in system (6); Section 5 performs

numerical simulations to illustrate the qualitative results; Section 6 makes some conclusions.

\section{Stability of the Disease-Free Equilibrium}

Let $(S, I)$ be an equilibrium of system (6). Then, $(S, I)$ must solve the following algebraic equations:

$$
\begin{gathered}
\sigma \phi+\sigma(1-\phi) \frac{r(1-p) I+S}{(1-p) I+S}-\beta S I-\eta S=0, \\
\sigma(1-\phi) \frac{(1-r)(1-p) I}{(1-p) I+S}+\beta S I-(\eta+\omega) I=0 .
\end{gathered}
$$

For convenience, we introduce the following threshold parameter:

$$
R_{0}=\frac{(1-\phi)(1-r)(1-p) \eta^{2}+\beta \sigma}{\eta(\eta+\omega)}
$$

Theorem 1. If $R_{0} \leq 1$, system (6) only has a disease-free equilibrium $E_{0}$ and if $R_{0}>1$, system (6) has a unique positive equilibrium $E^{*}$ except for the disease-free equilibrium $E_{0}$, where $E_{0}=(\sigma / \eta, 0), E^{*}=\left(S^{*}, I^{*}\right), S^{*}=\left(-a_{1}-\right.$ $\left.\sqrt{a_{1}^{2}-4 a_{0} a_{2}}\right) / 2 a_{2}, I^{*}=\left(\sigma(1-p)(1-r)(1-\phi)-S^{*}(\eta+\omega-\right.$ $\left.\left.\beta S^{*}\right)\right) /\left(\eta+\omega-\beta S^{*}\right)(1-p), a_{0}=\sigma(p-1)(\phi(r-1)-r)(\eta+\omega)$, $a_{1}=\eta^{2} p-\beta \sigma+\eta \omega p+\beta \sigma p+\omega^{2}+\eta \omega$, and $a_{2}=-\beta(\eta p+\omega)$.

By the transformation $x_{1}(t)=S(t)-(\sigma / \eta), x_{2}(t)=I(t)$, system (6) can be rewritten as the following equivalent form:

$$
\frac{\mathrm{d} x(t)}{\mathrm{d} t}=M x(t)+N x(t-\tau)+f(x(t)),
$$

where

$$
\begin{gathered}
M=\left(\begin{array}{cc}
-\eta & -(1-\phi)(p-1)(r-1) \eta \\
0 & (1-\phi)(1-r)(1-p) \eta-(\eta+\omega)
\end{array}\right), \quad N=\left(\begin{array}{cc}
0 & -\frac{\beta \sigma}{\eta} \\
0 & \frac{\beta \sigma}{\eta}
\end{array}\right), \quad x(t)=\left(\begin{array}{l}
x_{1}(t) \\
x_{2}(t)
\end{array}\right) \\
f\left(x_{1}(t), x_{2}(t)\right)=\left(\begin{array}{c}
-\beta x_{1}(t-\tau) x_{2}(t-\tau)+\frac{\eta^{2}(r-1)(p-1)(1-\phi) x_{2}(t)\left((p-1) x_{2}(t)-x_{1}(t)\right)}{\eta(p-1) x_{2}(t)-\eta x_{1}(t)-\sigma} \\
\beta x_{1}(t-\tau) x_{2}(t-\tau)-\frac{\eta^{2}(r-1)(p-1)(1-\phi) x_{2}(t)\left((p-1) x_{2}(t)-x_{1}(t)\right)}{\eta(p-1) x_{2}(t)-\eta x_{1}(t)-\sigma}
\end{array}\right) .
\end{gathered}
$$

It can be calculated that the characteristic equation of system (9) at the origin owns the following form:

$$
(\lambda+\eta)\left(\lambda-\eta(1-\phi)(1-r)(1-p)+\eta+\omega-\frac{\beta \sigma}{\eta} e^{-\lambda \tau}\right)=0 \text {. }
$$

Then, neglecting the time delay $\tau$, we have the following.
Theorem 2. If $R_{0}>1$, the disease-free equilibrium $E_{0}$ is unstable, while it is asymptotically stable if $R_{0}<1$.

Clearly, the origin is a nonhyperbolic equilibrium of system (9) if $R_{0}=1$ and $\tau=0$. To discuss its stability, we use the transformation as $x_{1}(t)=u(t)-((\eta+\omega) / \eta) v(t)$, $x_{2}(t)=v(t)$. Then, system (9) is changed into 


$$
\begin{gathered}
\frac{\mathrm{d} u(t)}{\mathrm{d} t}=-\eta u(t)+f_{1}(u(t), v(t)), \\
\frac{\mathrm{d} v(t)}{\mathrm{d} t}=f_{2}(u(t), v(t)),
\end{gathered}
$$

where

$$
\begin{aligned}
& f_{1}(u(t), v(t)) \\
& =\frac{-\omega v(t)}{\eta \sigma(\sigma-(\eta p+\omega) v(t)+\eta u(t))} \\
& \times(\eta \sigma(2 \eta(r+(1-r)(\phi+p(1-\phi)))+\omega-\eta) u(t) \\
& +\sigma\left(\eta^{2}\left(1+(1-r)(1-\phi)\left(p^{2}+1\right)\right)+\omega^{2}+2 \omega \eta\right. \\
& \times((1-r)(p(1-\phi)+\phi)+r)) v(t) \\
& -\eta^{2}(\eta((1-r)(p(1-\phi)+\phi)+r)+\omega) u(t)^{2} \\
& +\eta(\eta(1+p)+2 \omega) \\
& \times(\eta((r+p(1-r))(1-\phi)+\phi)+\omega) u(t) v(t) \\
& -(\eta+\omega)(\omega+\eta p) \\
& \left.\times(\eta((r+p(1-r))(1-\phi)+\phi)+\omega) v(t)^{2}\right), \\
& =\frac{-v(t)}{\sigma((\eta p+\omega) v(t)-\eta u(t)-\sigma)} \\
& \times(\sigma \eta(2 \eta(\phi(1-p)(1-r)+p(1-\phi)+r) \\
& -\eta+\omega) u(t) \\
& +\sigma\left(\omega^{2}+\eta^{2}\left(\left(p^{2}(1-r)+r\right)(1-\phi)+\phi\right)\right. \\
& +2 \eta \omega((1-\phi) \cdot(p(1-r)+r)+\phi)) v(t) \\
& -\eta^{2}(\eta((p(1-\phi)+\phi)(1-r)+r)+\omega) u(t)^{2} \\
& +\eta(\eta(1+p)+2 \omega) \\
& \times(\eta((1-\phi)(p(1-r)+r)+\phi)+\omega) u(t) v(t) \\
& -(\eta+\omega)(\omega+\eta p) \\
& \left.\times(\eta((1-\phi)(p(1-r)+r)+\phi)+\omega) v(t)^{2}\right) .
\end{aligned}
$$

By the existence theorem in the center manifold theory, see Wiggins [30] for details, there exists a center manifold for system (12), which can be locally expressed as follows:

$$
\begin{array}{r}
W^{c}(0)=\left\{(u, v) \in \mathbb{R}^{2} \mid u=h(v),\|v\|<\delta,\right. \\
h(0)=0, \operatorname{Dh}(0)=0 \mid \delta>0\},
\end{array}
$$

where $\delta$ is sufficiently small, $D h$ is the derivative of $h$ with respect to $v$.
Now, the first task is to compute the center manifold $W^{c}(0)$. For the purpose, we assume $h(v)$ has the form

$$
u=h(v)=h_{2} v^{2}+h_{3} v^{3}+h_{4} v^{4}+h_{5} v^{5}+\cdots,
$$

where $h_{i}, i=2, \ldots, 5$, are constants to be determined in the following. By the invariance of $W^{c}(0)$ under the dynamics of (12), $h(\cdot)$ satisfies

$$
D h \cdot f_{2}(h, v)+\eta h-f_{1}(h, v)=0 .
$$

Substituting (11) into (12), and then equating coefficients on each power of $v$ to zero, yields

$$
\begin{aligned}
& h_{2}=\frac{-\omega}{2 \sigma \eta^{2}}(2 \omega \eta((1-r)(\phi(1-p)+p)+r) \\
& \left.+\eta^{2}\left((1-\phi)\left(p^{2}(1-r)+r\right)+\phi\right)+\omega^{2}\right), \\
& h_{3}=\frac{-\omega}{4 \sigma^{2} \eta^{3}}\left(2 \phi^{2}(r-1)^{2}(p-1)^{2}(p+1)^{2}+2 \phi(1-r)\right. \\
& \times(1-p)\left(2 r(1-p)(1+p)^{2}+p^{2} \cdot(2 p+3)\right) \\
& -2\left(r(1-p)(1+p)^{2}\right. \\
& \left.+p\left(p^{2}+p-1\right)(r p-p-r)\right) \eta^{4} \\
& +\left(10 \phi^{2} \omega(1-r)^{2} \cdot(1+p)(1-p)^{2}+\phi(1-r)\right. \\
& \times(1-p)\left(20 p^{2}+13 p-1-20 r\left(1-p^{2}\right)\right) \\
& +\omega\left(10 p^{3}(r-1)^{2}+(1-r)(10 r+3) p^{2}\right. \\
& +2(1-r)(5 r-2) p+r(10 r-1))) \eta^{3} \\
& +\left(12 \phi^{2} \omega^{2}(1-r)^{2} \cdot(1-p)^{2}+\phi \omega^{2}\right. \\
& \times(1-r)(1-p)(29 p-24 r p+24 r-5) \\
& +\omega^{2}\left((r-1)(12 r-17) p^{2}\right) \\
& +24 r(1-r) p+(3 r+2)(4 r-1)) \eta^{2} \\
& +\left(12 \omega^{2}(1-r)(1-p) \phi\right. \\
& \left.\left.-\omega^{3}(12(1-r) \cdot(1-p)-11)\right) \eta+3 \omega^{4}\right),
\end{aligned}
$$$$
h_{4}=\frac{-1}{2 \sigma^{3} \eta}\left(\left(2 h_{2} p^{2} \sigma((1-r)(1-\phi)(1-p)-p r)\right.\right.
$$$$
\left.-(1-r)(1-\phi)(1-p) p^{3} \omega\right) \eta^{3}
$$$$
+\left(6 h_{2} \sigma p \omega(1-r)(1-p)(1-\phi)\right.
$$ 


$$
\begin{aligned}
& +2 h_{2}^{2} \sigma^{2}(1-2(1-r)(1-p)(1-\phi)) \\
& -3 h_{3} \sigma^{2} \cdot\left((1-\phi)\left(p^{2}(1-r)+r\right)+r\right) \\
& \left.\quad-3 p^{2} \omega^{2}(1-r)(1-\phi)(1-p)\right) \eta^{2} \\
& -\left(h_{3} \sigma^{2} \omega(8(1-r) \cdot(1-p)(1-\phi)-7)\right. \\
& -4 h_{2} \sigma \omega^{2}(1-r)(1-p)(1-\phi) \\
& \left.+2 h_{2}^{2} \sigma^{2} \omega^{2}-3 p \omega^{3}(1-r) \cdot(1-p)(1-\phi)\right) \eta \\
& \left.-4 h_{3} \sigma^{2} \omega^{2}-(1-r)(1-r)(1-\phi)\right) .
\end{aligned}
$$

On substituting (18) into the second equation of system (12), we obtain the following equation on the center manifold $W^{c}(0)$ :

$$
\frac{\mathrm{d} v(t)}{\mathrm{d} t}=d_{2} v(t)^{2}+d_{3} v(t)^{3}+\cdots
$$

where

$$
\begin{aligned}
& d_{2}=\frac{-2}{\sigma} \\
& \times\left(\left(\left(p^{2}-1\right)(1-\phi)(1-r)-1\right) \eta^{2}\right. \\
& \left.+2 \omega(1-(1-p)(1-r)(1-\phi)) \eta+\omega^{2}\right), \\
& d_{3}=\frac{-3}{\eta \sigma^{2}}\left(2 p^{2}(r-1)(\phi-1)(p-1) \eta^{4}\right. \\
& +\omega\left(2 \phi^{2}(r-1)^{2}(p-1)^{2}(p+1)+\phi(1-p)\right. \\
& \cdot\left(4 r^{2}(p-1)(p+1)-r\left(8 p^{2}+5 p-5\right)\right. \\
& \left.+4 p^{2}+5 p-1\right) \\
& +p\left(2 p^{2}+3 p-1\right)-r \cdot\left(4 p^{2}+p-6\right) \\
& \left.+2 r^{2}(1-p)^{2}(1+p)\right) \eta^{3} \\
& +\omega^{2}\left(\phi ^ { 2 } \left(4(1-r)^{2}+p(p-2)\right.\right. \\
& \left.+p^{2} r(r-2)+p r(p r+4)\right) \\
& +\phi(1-p) \\
& \times\left(9 p+1-r(17 p-7)-8 r^{2}(1-p)\right) \\
& +5 p^{2}-2+(1-p)(1+9 p) \\
& \left.+4 r^{2}(1-p)^{2}\right) \eta^{2} \\
& \left.+\omega^{3}(3-4(1-p)(1-r)(1-\phi)) \eta+\omega^{4}\right) \text {. }
\end{aligned}
$$

Therefore, by using the center manifold theorem [30], we have the following results.
Theorem 3. If $R_{0}=1$, the disease-free equilibrium $E_{0}$ is unstable since $d_{2}<0$.

It can be seen that (11) always has a negative root $-\eta$. To investigate the stability of the disease-free equilibrium when $\tau>0$, we reduce (11) to the following

$$
\lambda+p_{1}+q_{1} e^{-\lambda \tau}=0,
$$

where $p_{1}=\eta+\omega-\eta(1-\phi)(1-r)(1-p), q_{1}=-(\beta \sigma / \eta)$.

Suppose that $\lambda=i y, y>0$ is a root of (20), then $y$ satisfies

$$
p_{1}+q_{1} \cos (y \tau)=0, \quad y-q_{1} \sin (y \tau)=0 .
$$

Eliminating the trigonometric functions yields

$$
\frac{y^{2}}{q_{1}^{2}}=\frac{q_{1}^{2}-p_{1}^{2}}{q_{1}^{2}}
$$

It can be computed that

$$
q_{1}^{2}-p_{1}^{2}=-\frac{\eta+\omega}{q_{1}^{2}}\left(\left(1-R_{0}\right)^{2}+\frac{2 \beta \sigma}{\eta(\eta+\omega)}\left(1-R_{0}\right)\right) .
$$

Then (22) leads to a contradiction if $R_{0}<1$, and we have the following results.

Theorem 4. If $R_{0}<1$, the disease-free equilibrium $E_{0}$ is asymptotically stable for any $\tau>0$. If $R_{0}>1+(\beta \sigma / \eta(\eta+\omega))$, $E_{0}$ is unstable for any $\tau>0$.

It can be seen that $q_{1}^{2}-p_{1}^{2}>0$ if $1<R_{0}<1+(\beta \sigma / \eta(\eta+$ $\omega)$ ), which means that (22) has a unique positive root $y_{0}=$ $\sqrt{q_{1}^{2}-p_{1}^{2}}$. That is, there is a single pair of purely imaginary roots $\pm i y_{0}$ of $(20)$. Let $\tau_{k}=\left(1 / y_{0}\right) \arccos (-p 1 / q 1)+2 k \pi / y_{0}$, $k=1,2,3, \ldots$.

Theorem 5. If $1<R_{0}<1+(\beta \sigma / \eta(\eta+\omega))$, the disease-free equilibrium $E_{0}$ is unstable for any $0<\tau<\tau_{0}$.

Theorem 6. If $\tau=\tau_{k}$ and $1<R_{0}<1+(\beta \sigma / \eta(\eta+\omega))$, system (6) undergoes Hopf bifurcations at the disease-free equilibrium $E_{0}$.

Proof. Differentiating (20) with respect to $\tau$ yields

$$
\left(1-q_{1} \tau e^{-\lambda \tau}\right) \frac{\mathrm{d} \lambda}{\mathrm{d} \tau}=\lambda q_{1} e^{-\lambda \tau}
$$

which gives

$$
\left(\frac{\mathrm{d} \lambda}{\mathrm{d} \tau}\right)^{-1}=-\frac{\tau}{-\lambda}-\frac{1}{\lambda\left(\lambda+p_{1}\right)} .
$$

When $\lambda= \pm i y_{0}$ and $\tau=\tau_{k}$, we obtain

$$
\begin{aligned}
\left.\left(\frac{\mathrm{d} \mathfrak{R e}\{\lambda\}}{\mathrm{d} \tau}\right)^{-1}\right|_{\lambda= \pm i y_{0}, \tau=\tau_{k}} & =\left.\operatorname{Re}\left(\frac{\mathrm{d} \lambda}{\mathrm{d} \tau}\right)^{-1}\right|_{\lambda= \pm i y_{0}, \tau=\tau_{k}} \\
& =\frac{y_{0}^{2}}{y_{0}^{4}+p_{1}^{2} y_{0}^{2}}>0 .
\end{aligned}
$$

The transversality condition for Hopf bifurcations holds. Then system (6) undergoes Hopf bifurcations at the diseasefree equilibrium $E_{0}$ when $\tau=\tau_{k}$. 


\section{Normal Forms on the Center Manifold for a Simple Zero Eigenvalue}

It can be obtained that zero is a simple eigenvalue of $R_{0}=$ 1 and $\tau \neq 1 / q_{1}$. In this section, we refer the reader to Hale and Lunel [31] for notation and general results on the theory of retarded functional differential equations (RFDES). To determine the dynamic properties of the disease-free equilibrium $E_{0}$ with $R_{0}=1, \tau>0$, we have to compute the normal forms on the center manifold. The method we use is based on the center manifold reduction and normal form theory; see Faria and Magalhaes [32, 33]. In the following, we shall compute the normal form of model (6) associated with the zero eigenvalue.

For convenience, we rescale the time by $t \rightarrow t / \tau$ to normalize the delay of system (9) and obtain

$$
\begin{aligned}
\frac{\mathrm{d} u_{1}(t)}{\mathrm{d} t}=\tau[ & -\eta u_{1}(t)-\eta(1-\phi)(p-1)(r-1) u_{2}(t) \\
& -\frac{\beta \sigma}{\eta} u_{2}(t-1)-\beta u_{1}(t-1) u_{2}(t-1) \\
& +\left(\eta^{2}(r-1)(p-1)(1-\phi)\right. \\
& \left.\times\left(u_{2}(t)(p-1)-u_{1}(t)\right) u_{2}(t)\right) \\
& \left.\times\left(\eta\left((p-1) u_{2}(t)-u_{1}(t)\right)-\sigma\right)^{-1}\right] \\
\frac{\mathrm{d} u_{2}(t)}{\mathrm{d} t}=\tau[ & (\eta(1-\phi)(1-r)(1-p)-(\eta+\omega)) u_{2}(t) \\
& +\frac{\beta \sigma}{\eta} u_{2}(t-1)+\beta u_{1}(t-1) u_{2}(t-1)
\end{aligned}
$$

$$
\begin{aligned}
& -\left(\eta^{2}(1-\phi)(1-r)(1-p)\right. \\
& \left.\quad \times\left(u_{2}(t)(p-1)-u_{1}(t)\right) u_{2}(t)\right) \\
& \left.\times\left(\eta\left((p-1) u_{2}(t)-u_{1}(t)\right)-\sigma\right)^{-1}\right] .
\end{aligned}
$$

Let $\mathscr{C}=\mathscr{C}\left([-1,0], \mathbb{R}^{2}\right)$ be the Banach space of continuous functions from $[-1,0]$ into $\mathbb{R}^{2}$ with supremum norm. We define $z_{t} \in \mathscr{C}$, as $z_{t}(\theta)=z(t+\theta), \theta \in[-1,0]$. Using the perturbation: of $\beta=\beta_{0}+\epsilon$, where $\beta_{0}=(\eta / \sigma)(\eta+w-(1-$ $\phi)(1-r)(1-p) \eta)$, system $(27)$ can be written as the functional differential equation

$$
\dot{z}(t)=L(\epsilon)\left(z_{t}\right)+F\left(z_{t}, \epsilon\right)
$$

where $\epsilon \in V$ is considered as a parameter, $V$ is a neighborhood of zero in space of real numbers, $L: \mathscr{C} \times V \rightarrow \mathbb{R}^{2}$ is a parameterized family of bounded linear operators, and $F$ : $\mathscr{C} \times V \rightarrow \mathbb{R}^{2}$ is a function with $F(0, \epsilon)=0, \partial F(0, \epsilon) / \partial z=0$ for all $\epsilon \in \mathbb{R}$, and they have the following respective forms:

$$
\begin{aligned}
L(\epsilon)(\varphi) & =L_{0} \varphi+L_{1}(\epsilon) \varphi, F(\varphi, \epsilon) \\
& =F_{2}(\varphi, \epsilon)+F_{3}(\varphi, \epsilon)+\text { h.o.t }
\end{aligned}
$$

where h.o.t stands for the higher-order terms, $\varphi=\left(\varphi_{1}, \varphi_{2}\right)^{T}$, and

$$
\left.\begin{array}{c}
F_{2}(\varphi, \epsilon)=\tau\left(\begin{array}{l}
\frac{\eta^{2}}{\sigma}(r-1)(p-1)(1-\phi)\left((p-1) \varphi_{2}^{2}(0)-\varphi_{1}(0) \varphi_{2}(0)\right)-\beta_{0} \varphi_{1}(-1) \varphi_{2}(-1) \\
\frac{\eta^{2}}{\sigma}(1-r)(1-p)(\phi-1)\left((p-1) \varphi_{2}^{2}(0)-\varphi_{1}(0) \varphi_{2}(0)\right)+\beta_{0} \varphi_{1}(-1) \varphi_{2}(-1)
\end{array}\right), \\
F_{3}(\varphi, \epsilon)=\tau\left(\begin{array}{c}
-\epsilon_{0} \varphi_{1}(-1) \varphi_{2}(-1)+\frac{\eta^{3}}{\sigma^{2}}(r-1)(p-1)(1-\phi) \varphi_{2}(0) \\
\cdot\left(\varphi_{1}(0)-(p-1) \varphi_{2}(0)\right)\left((p-1) \varphi_{2}(0)-\varphi_{1}(0)\right) \\
\epsilon_{0} \varphi_{1}(-1) \varphi_{2}(-1)+\frac{\eta^{3}}{\sigma^{2}}(r-1)(p-1)(\phi-1) \varphi_{2}(0) \\
\cdot\left(\varphi_{1}(0)-(p-1) \varphi_{2}(0)\right)\left((p-1) \varphi_{2}(0)-\varphi_{1}(0)\right)
\end{array}\right), \\
L_{0} \varphi=\tau\left(\begin{array}{c}
-\eta(1-\phi)(1-p)(r-1) \varphi_{2}(0) \\
-\frac{\beta_{0} \sigma}{\eta} \varphi_{2}(-1)-\eta \varphi_{1}(0) \\
(\eta(-\phi)(1-r)(1-p)-\eta-\omega) \\
\varphi_{2}(0)+\frac{\beta_{0} \sigma}{\eta} \varphi_{2}(-1)
\end{array}\right), \\
L_{1}(\epsilon) \varphi=\tau\left(\begin{array}{c}
-\frac{\sigma}{\eta} \epsilon \varphi_{2}(-1) \\
\frac{\sigma}{\eta} \epsilon \varphi_{2}(-1)
\end{array}\right)
\end{array}\right) .
$$

From the Riesz representation theorem the linear map $L$ can be expressed in integral form as follows:

$$
L(\epsilon)(\varphi)=\int_{-1}^{0} \mathrm{~d} \eta_{\epsilon}(\theta) \varphi(\theta)
$$


where $\eta_{\epsilon}(\cdot)$ is a bounded variation function on $[-1,0]$ and can be defined as

$$
\eta_{\epsilon}(\theta)=\tau M \delta(\theta)+\tau N(\epsilon) \delta(\theta+1),
$$

$\delta(\cdot)$ is the Dirac delta function, and $N(\epsilon)=\left(\begin{array}{cc}0 & -\left(\left(\beta_{0}+\epsilon\right) \sigma / \eta\right) \\ 0 & \left(\beta_{0}+\epsilon\right) \sigma / \eta\end{array}\right)$.

Let $\mathbb{R}^{2 *}$ be the 2-dimensional vector space of row vectors and denote $\mathscr{C}^{*}=\mathscr{C}\left([-1,0], \mathbb{R}^{2 *}\right)$. We define the adjoint bilinear form on $\mathscr{C}^{*} \times \mathscr{C}$ as follows:

$$
\begin{aligned}
\langle\psi(s), \varphi(\theta)\rangle= & \psi(0) \varphi(0) \\
& -\int_{-1}^{0} \int_{0}^{\theta} \psi(\xi-\theta) \mathrm{d} \eta_{\epsilon}(\theta) \varphi(\xi) \mathrm{d} \xi,
\end{aligned}
$$

where $\psi=\left(\psi_{1}, \psi_{2}\right) \in \mathscr{C}^{*}$ and $\varphi=\left(\varphi_{1}, \varphi_{2}\right)^{T} \in \mathscr{C}$.

Let $A(\epsilon)$ be the infinitesimal generator of the flow for the linear system $\dot{z}(t)=L(\epsilon) z_{t}$, with spectrum $\sigma[A(\epsilon)]$. The adjoint operator $A^{*}(\epsilon)$ is defined as the infinitesimal generator for the solution operator of the adjoint equation in $\mathscr{C}^{*}$

$$
\dot{w}(t)=-\int_{-1}^{0} w(t-\theta) \mathrm{d} \eta_{\epsilon}(\theta) .
$$

It is well known that the eigenvalues of $A(\epsilon)$ with zero real parts play an important role in the bifurcation theory of RFDES. Let $A_{0}=A(0)$ and denote all of its singular eigenvalues by the set $\Lambda_{0}$; that is,

$$
\Lambda_{0}=\left\{\lambda \in \sigma\left(A_{0}\right) \mid \mathfrak{R e} \lambda=0\right\} .
$$

According to the discussion in Section 2, we have $\Lambda_{0}=\{0\}$.

Using the formal adjoint theory for FDEs in Hale and Lunel [31], the phase space $\mathscr{C}$ can be decomposed by $\Lambda_{0}$ as $\mathscr{C}=P \oplus Q$, where $P$ is the generalized eigenspace associated with the eigenvalues in $\Lambda_{0}, Q=\{\varphi \in \mathscr{C} \mid\langle\psi, \varphi\rangle=$ 0 for all $\left.\psi \in P^{*}\right\}$, and the dual space $P^{*}$ is the generalized eigenspace for $A^{*}(0)$ associated with the eigenvalues in $\Lambda_{0}$. In particular, we consider bases for $P$ and $P^{*}$ denoted by $\Phi$ and $\Psi$, respectively, and satisfying $\langle\Psi(s), \Phi(\theta)\rangle=1$. Then, we can choose $\Phi$ and $\Psi$ as follows:

$$
\begin{gathered}
\Phi(\theta)=(\eta+\omega,-\eta)^{T}, \quad-1 \leq \theta \leq 0, \\
\Psi(s)=(0, k), \quad 0 \leq s \leq 1,
\end{gathered}
$$$$
\text { where } k=-\frac{1}{\eta+\beta_{0} \sigma \tau} \text {. }
$$

Let $B=0$. Then, the following equations satisfy simultaneously

$$
\dot{\Phi}=\Phi B, \quad \dot{\Psi}=-B \Psi .
$$

As shown in Faria and Magalhaes [32, 33], an appropriate phase space for considering normal forms of (28) is the Banach space $\mathbf{B C}$ of functions from $[-1,0]$ into $\mathbb{R}^{2}$ which are uniformly continuous on $[-1,0)$ with a jump discontinuity at 0 . Then, the elements of $\mathbf{B C}$ have the form $\varphi+X_{0} \rho$, where $\varphi \in \mathscr{C}, \rho \in \mathbb{R}^{2}$, and

$$
X_{0}(\theta)= \begin{cases}I, & \theta=0, \\ 0, & -1 \leq \theta<0,\end{cases}
$$

so that BC is identified with $\mathscr{C} \times \mathbb{R}^{2}$ with the norm $\left|\varphi+X_{0} \rho\right|=$ $|\varphi|_{C}+|\rho|_{\mathbb{R}^{2}}$.

Let $\pi: \mathbf{B C} \rightarrow P$ denote the projection

$$
\pi\left(\varphi+X_{0} \rho\right)=\Phi(\langle\Psi, \varphi\rangle+\Psi(0) \rho), \quad \varphi \in \mathscr{C}, \rho \in \mathbb{R}^{2},
$$

and then the decomposition $\mathscr{C}=P \oplus Q$ yields a decomposition of $\mathbf{B C}$ by $\Lambda_{0}$ as the topological direct sum $\mathbf{B C}=$ $P \oplus \operatorname{Ker} \pi$ with the property $Q \subset \operatorname{Ker} \pi$, where $Q$ is an infinitedimensional complementary subspace of $P$ and $\mathscr{C}$ as shown above. Now, we decompose $z_{t} \in \mathscr{C}^{1}$ in (28) as $z_{t}=\Phi x(t)+y$, where $x(t) \in \mathbb{R}$ and $y \in Q^{1}=Q \cap \mathscr{C}^{1}$, and $\mathscr{C}^{1}$ is the subset of $\mathscr{C}$ consisting of continuously differentiable functions. We rewrite system (28) as

$$
\dot{z}(t)=L_{0} z_{t}+\left(L(\epsilon)-L_{0}\right) z_{t}+F\left(z_{t}, \epsilon\right),
$$

and then, under the composition $z_{t}=\Phi x(t)+y$, system (28) can be decomposed as a system of ODEs in $\mathbb{R} \times \operatorname{Ker} \pi$ as follows:

$$
\begin{aligned}
\dot{x}=B x+ & \Psi(0)\left(\left(L(\epsilon)-L_{0}\right)(\Phi x+y)+F(\Phi x+y, \epsilon)\right), \\
\dot{y}= & A_{\mathrm{Q}_{1}} y+(I-\pi) X_{0} \\
& \times\left(\left(L(\epsilon)-L_{0}\right)(\Phi x+y)+F(\Phi x+y, \epsilon)\right),
\end{aligned}
$$

where $A_{\mathrm{Q}_{1}}=\dot{y}+X_{0}(L(y)-\dot{y}(0))$ is an operator from $Q^{1}$ into Ker $\pi$.

Considering the Taylor expansion of the functions to the right of (41), we have

$$
\begin{gathered}
\dot{x}=B x+\sum_{j \geq 2} \frac{1}{j !} f_{j}^{1}(x, y, \epsilon), \\
\dot{y}=A_{Q_{1}} y+\sum_{j \geq 2} \frac{1}{j !} f_{j}^{2}(x, y, \epsilon),
\end{gathered}
$$

where

$$
\begin{aligned}
& \frac{1}{j !} f_{j}^{1}(x, y, \epsilon) \\
& =\Psi(0)\left[\frac{1}{(j-1) !} L_{j-1}(\epsilon)(\Phi x+y)+\frac{1}{j !} F_{j}(\Phi x+y, \epsilon)\right], \\
& \frac{1}{j !} f_{j}^{2}(x, y, \epsilon) \\
& =(I-\pi) X_{0}\left[\frac{1}{(j-1) !} L_{j-1}(\epsilon)(\Phi x+y)+\frac{1}{j !} F_{j}(\Phi x+y, \epsilon)\right] .
\end{aligned}
$$

It can be obtained that 


$$
\begin{aligned}
\frac{1}{2 !} f_{2}^{1}(x, y, \epsilon)=k \tau & {\left[\frac{\epsilon \sigma}{\eta}\left(y_{2}(-1)-\eta x\right)+\frac{\eta^{2}}{\sigma}(1-\phi)(1-r)(1-p)\right.} \\
& \left.\times\left((p-1)\left(\eta x-y_{2}(-1)\right)^{2}+\left(\eta x-y_{2}(0)\right)\left((\eta+\omega) x+y_{1}(0)\right)\right)-\beta_{0}\left((\eta+\omega) x+y_{1}(-1)\right)\left(\eta x+y_{2}(-1)\right)\right] \\
\frac{1}{2 !} f_{2}^{2}(x, y, \epsilon)=(I-\pi) X_{0} & \cdot\left(\begin{array}{c}
-\frac{\eta^{2} \tau}{\sigma}(1-\phi)(1-r)(1-p)\left[(p-1)\left(y_{2}(0)-\eta x\right)^{2}-\left((\eta+\omega) x+y_{1}(0)\right)\left(y_{2}(0)-\eta x\right)\right] \\
\frac{\epsilon \sigma \tau}{\eta}\left(y_{2}(-1)-\eta x\right)+\beta_{0} \tau\left((\eta+\tau) x+y_{1}(-1)\right)\left(y_{2}(-1)-\eta x\right) \\
-\frac{\epsilon \sigma \tau}{\eta}\left(y_{2}(-1)-\eta x\right)-\beta_{0} \tau\left((\eta+\tau) x+y_{1}(-1)\right)\left(y_{2}(-1)-\eta x\right) \\
-\frac{\eta^{2} \tau}{\sigma}(1-\phi)(1-r)(1-p)\left[(p-1)\left(y_{2}(0)-\eta x\right)^{2}-\left((\eta+\omega) x+y_{1}(0)\right)\left(y_{2}(0)-\eta x\right)\right]
\end{array}\right) .
\end{aligned}
$$

As for autonomous ODEs in $\mathbb{R}^{2}$, the normal forms are obtained by a recursive process of changes of variables. At a step $j$, the terms of order $j \geq 2$ are computed from the terms of the same order and from the terms of lower orders already computed in previous steps. Assume that steps of orders $2,3, \ldots, j-1$ have already been performed; this leads to

$$
\begin{gathered}
\dot{x}=B x+\sum_{l \geq 2}^{j-1} \frac{1}{l !} g_{l}^{1}(x, y, \epsilon)+\frac{1}{j !} \widetilde{f}_{j}^{1}(x, y, \epsilon)+\text { h.o.t, } \\
\dot{y}=A_{\mathrm{Q}_{1}} y+\sum_{l \geq 2}^{j-1} \frac{1}{l !} g_{l}^{2}(x, y, \epsilon)+\frac{1}{j !} \widetilde{f}_{j}^{2}(x, y, \epsilon)+\text { h.o.t. }
\end{gathered}
$$

Following the algorithm of Faria and Magalhaes [32, 33] at step $j$, using a change of variables of the form

$$
(x, y)=(\widehat{x}, \widehat{y})+U_{j}(\widehat{x}, \epsilon) \equiv(\widehat{x}, \widehat{y})+\left[U_{j}^{1}(\widehat{x}, \epsilon), U_{j}^{2}(\widehat{x}, \epsilon)\right],
$$

where $x, \widehat{x} \in \mathbb{R}, y, \hat{y} \in Q^{1}$, and $U_{j}^{1}: \mathbb{R}^{2} \rightarrow \mathbb{R}, U_{j}^{2}: \mathbb{R}^{2} \rightarrow Q^{1}$ are homogeneous polynomials of degree $j$ in $\widehat{x}$ and $\epsilon$, after dropping the hats for simplification of notations, system (42) can be put into the normal form

$$
\begin{gathered}
\dot{x}=B x+\sum_{j \geq 2} \frac{1}{j !} g_{j}^{1}(x, y, \epsilon), \\
\dot{y}=A_{Q^{1}} y+\sum_{j \geq 2} \frac{1}{j !} g_{j}^{2}(x, y, \epsilon),
\end{gathered}
$$

where

$$
\begin{gathered}
g_{j}^{1}(x, y, \epsilon)=\tilde{f}_{j}^{1}(x, y, \epsilon)-\left[D_{x} U_{j}^{1}(x, \epsilon) B x-B U_{j}^{1}(x)\right], \\
g_{j}^{2}(x, y, \epsilon)=\widetilde{f}_{j}^{2}(x, y, \epsilon)-\left[D_{x} U_{j}^{2}(x, \epsilon) B x-A_{\mathrm{Q}^{1}} U_{j}^{2}(x)\right] .
\end{gathered}
$$

It can be verified that system (28) satisfies nonresonance conditions since $\Lambda_{0}=\{0\}$; see Faria and Magalhaes [32]. Then, the locally invariant manifold of system (28) tangent to $P$ at zero must be $y=0$ and the flow on this manifold is given by 1-dimensional ODE as follows:

$$
\dot{x}=B x+\frac{1}{2 !} g_{2}^{1}(x, 0, \epsilon)+\frac{1}{3 !} g_{3}^{1}(x, 0, \epsilon)+\text { h.o.t. }
$$

The nonlinear terms in (47) are in normal form in the classical sense with respect to matrix $B$. In applications, $g_{j}^{1}(x, 0, \epsilon)$ usually can be determined by the following procedure.

Definition 7. For $j \geq 2$, let $M_{j}$ denote the operator defined in $V_{j}\left(\mathbb{R}^{2} \times \operatorname{Ker} \pi\right)$, with values in the same place, by

$$
\begin{gathered}
M_{j}\left(h_{1}, h_{2}\right)=\left(M_{j}^{1} h_{1}, M_{j}^{2} h_{2}\right), \\
\left(M_{j}^{1} h_{1}\right)(x, \epsilon)=D_{x} h_{1}(x, \epsilon) B x-B h_{1}(x, \epsilon), \\
\left(M_{j}^{2}\right)(x, \epsilon)=D_{x} h_{2}(x, \epsilon) B x-A_{\mathrm{Q}_{1}}\left[h_{2}(x, \epsilon)\right],
\end{gathered}
$$

with domain $D\left(M_{j}\right)=V_{j}^{2}\left(\mathbb{R}^{2}\right) \times V_{j}^{2}\left(Q^{1}\right)$. Here, we use the notation $V_{j}^{2}(Y)$ to denote the space of homogeneous polynomials of degree $j$ in 2 variables $(x, \epsilon) \in \mathbb{R}^{2}$, with coefficients in a Banach space $Y$.

According to Faria and Magalhaes [32, 33], we have

$$
\begin{gathered}
U_{j}(x)=M_{j}^{-1} P_{I, j} \tilde{f}_{j}(x, 0, \epsilon) \in \operatorname{Ker}\left(M_{j}\right)^{c}, \\
g_{j}^{1}(x, 0, \epsilon)=\left(I-P_{I, j}\right) \tilde{f}_{j}^{1}(x, 0, \epsilon) \in \operatorname{Im}\left(M_{j}^{1}\right)^{c},
\end{gathered}
$$

where $P_{I, j}=\left(P_{I, j}^{1}, P_{I, j}^{2}\right)$ is the projection of $V_{j}^{2}\left(\mathbb{R}^{2}\right) \times V_{j}^{2}(\operatorname{Ker} \pi)$ on $\operatorname{Im}\left(M_{j}^{1}\right) \times \operatorname{Im}\left(M_{j}^{2}\right)$. 
Since $B=0$, it can be checked that $\left[\operatorname{Im}\left(M_{2}^{1}\right)\right]^{2}=$ $\operatorname{span}\left\{x^{2}, x \epsilon, \epsilon^{2}\right\}$. Then, we have

$$
\begin{aligned}
\frac{1}{2} g_{2}^{1}(x, 0, \epsilon) & \\
= & k \tau\left(-\epsilon \sigma x+\frac{\eta^{2}}{\sigma}(1-\phi)(1-r)(1-p)\right. \\
& \left.\quad \times\left((p-1) \eta^{2} x^{2}+\eta(\eta+\omega) x^{2}-\beta_{0} \eta(\eta+\omega) x^{2}\right)\right) \\
= & k \tau\left(-\epsilon \sigma x+k_{1} x^{2}\right),
\end{aligned}
$$

where $k_{1}=\left(\eta^{3} / \sigma\right)(1-\phi)(1-r)(1-p)\left((p \eta+\omega)-\beta_{0}(\eta+\omega)\right)$. Further the normal form of (41) on the invariant local center manifold $y=0$ is given by

$$
\dot{x}=k \tau\left(-\epsilon \sigma x+k_{1} x^{2}\right)+\text { h.o.t. }
$$

Furthermore, if $k_{1}=0$, we have to compute $g_{3}^{1}(x, 0, \epsilon)$. It can be obtained that $\operatorname{Ker}\left(M_{2}^{1}\right)^{c}=0$, and

$$
U_{2}(x, \epsilon)=M_{2}^{-1} P_{I, 2}\left(\begin{array}{l}
f_{2}^{1}(x, 0, \epsilon) \\
f_{2}^{2}(x, 0, \epsilon)
\end{array}\right)=\left(\begin{array}{c}
0 \\
h_{2}(\theta)(x, \epsilon)
\end{array}\right),
$$

where $h_{2}(\theta)(x, \epsilon)=\left(h_{2}^{(1)}(\theta)(x, \epsilon), h_{2}^{(2)}(\theta)(x, \epsilon)\right)^{T}$ is the unique solution in $U_{2}^{2}\left(Q_{1}\right)$ of the equation

$$
M_{2}^{2} h_{2}(\theta)(x, \epsilon)=(I-\pi) X_{0}\left(\begin{array}{c}
2 \epsilon \sigma \tau x \\
-2 \epsilon \sigma \tau x
\end{array}\right) .
$$

Let

$$
\begin{aligned}
& h_{2}^{(i)}(\theta)(x, \epsilon)=\sum_{|q|=2} h_{2, q}^{(i)}(\theta)(x, \epsilon)^{q} \\
&=h_{220}^{(i)}(\theta) x^{2}+h_{211}^{(i)}(\theta) x \epsilon+h_{202}^{(i)}(\theta) \epsilon^{2}, \\
& i=1,2 .
\end{aligned}
$$

By (55), we get

$$
\begin{aligned}
& M h_{2}(0)(x \epsilon)+N(0) h_{2}(-1)(x, \epsilon)-\dot{h}_{2}(0)(x, \epsilon) \\
& =\left(\begin{array}{c}
2 \sigma \tau \epsilon x \\
-2 \epsilon \tau \epsilon x
\end{array}\right), \\
& \left(\begin{array}{c}
\dot{h}_{220}^{(1)}(\theta) x^{2}+\dot{h}_{211}^{(1)}(\theta) x \epsilon+\dot{h}_{201}^{(1)}(\theta) \epsilon^{2} \\
\dot{h}_{220}^{(2)}(\theta) x^{2}+\dot{h}_{211}^{(2)}(\theta) x \epsilon+\dot{h}_{201}^{(2)}(\theta) \epsilon^{2}
\end{array}\right) \\
& +\left(\begin{array}{c}
2 k(\eta+\omega) \tau \epsilon x \\
-2 k \eta \sigma \tau \epsilon x
\end{array}\right)=0,
\end{aligned}
$$

which leads to

$$
\begin{gathered}
-\eta \tau h_{220}^{(1)}(0)-\tau\left(\eta+\omega-\frac{\beta_{0} \sigma}{\eta}\right) h_{220}^{(2)}(0) \\
-\frac{\beta_{0} \sigma \tau}{\eta} h_{220}^{(2)}(-1)-\dot{h}_{220}^{(1)}(0)=0, \\
-\eta \tau h_{211}^{(1)}(0)-\tau\left(\eta+\omega-\frac{\beta_{0} \sigma}{\eta}\right) h_{211}^{(2)}(0) \\
-\frac{\beta_{0} \sigma \tau}{\eta} h_{211}^{(2)}(-1)-\dot{h}_{211}^{(1)}(0)=2 \sigma \tau, \\
-\eta \tau h_{202}^{(1)}(0)-\tau\left(\eta+\omega-\frac{\beta_{0} \sigma}{\eta}\right) h_{202}^{(2)}(0) \\
-\frac{\beta_{0} \sigma \tau}{\eta} h_{202}^{(2)}(-1)-\dot{h}_{202}^{(1)}(0)=0, \\
-\frac{\beta_{0} \sigma \tau}{\eta} h_{220}^{(2)}(0)+\frac{\beta_{0} \sigma \tau}{\eta} h_{220}^{(2)}(-1)-\dot{h}_{220}^{(1)}(0)=0, \\
-\frac{\beta_{0} \sigma \tau}{\eta} h_{211}^{(2)}(0)+\frac{\beta_{0} \sigma \tau}{\eta} h_{211}^{(2)}(-1)-\dot{h}_{211}^{(1)}(0)=-2 \sigma \tau, \\
-\frac{\beta_{0} \sigma \tau}{\eta} h_{202}^{(2)}(0)+\frac{\beta_{0} \sigma \tau}{\eta} h_{202}^{(2)}(-1)-\dot{h}_{202}^{(1)}(0)=0 .
\end{gathered}
$$

After computation, we arrive at

$$
\begin{gathered}
h_{220}(\theta)=\left(\begin{array}{l}
0 \\
0
\end{array}\right), \quad h_{202}(\theta)=\left(\begin{array}{l}
0 \\
0
\end{array}\right), \\
h_{211}(\theta)=\left(\begin{array}{l}
c_{1}+a \theta \\
c_{2}+b \theta
\end{array}\right),
\end{gathered}
$$

where $c_{1}=-\left(\beta_{0} \tau \sigma(\eta+\omega) b / 2 \eta\left(\eta+\beta_{0} \sigma \tau\right)\right)+(2 k \sigma \omega / \eta), c_{2}=$ $\beta_{0} \sigma \tau b / 2\left(\eta+\beta_{0} \sigma \tau\right), a=-2 k(\eta+\omega) \sigma \tau$, and $b=2 k \eta \sigma \tau$.

Then, we have

$$
U_{2}(x, \epsilon)=\left(\begin{array}{c}
0 \\
a \theta+c_{1} \\
b \theta+c_{2}
\end{array}\right) x \epsilon .
$$

Substituting the change of variables $(x, y)=(\widehat{x}, \hat{y})+$ $(1 / 2) U_{2}(\widehat{x}, \epsilon)$ into (48) and dropping the hats, we have 


$$
\begin{aligned}
& \frac{1}{3 !} \widetilde{f}_{3}^{1}(x, 0, \epsilon)=\frac{\sigma \tau \epsilon}{\eta} \Psi(0)\left(\begin{array}{c}
\left(b_{2}-c_{2}\right) x \epsilon \\
\left(c_{2}-b\right) x \epsilon
\end{array}\right)+\tau \Psi(0)\left(\begin{array}{c}
\frac{\eta^{5}}{\sigma^{2}}(r-1)(p-1)(1-\phi)(\omega+p \eta)^{2} x^{3}+\eta(\eta+\omega) \epsilon x^{2} \\
-\frac{\eta^{5}}{\sigma^{2}}(r-1)(p-1)(1-\phi)(\omega+p \eta)^{2} x^{3}-\eta(\eta+\omega) \epsilon x^{2}
\end{array}\right) \\
& =k \tau\left(\frac{\left(c_{2}-b\right) \sigma}{\eta} \epsilon^{2} x-\eta(\eta+\omega) \epsilon x^{2}-\frac{\eta^{5}}{\sigma^{2}}(r-1)(p-1)(1-\phi)(\omega+p \eta)^{2} x^{3}\right) .
\end{aligned}
$$

Since $\left[\operatorname{Im}\left(M_{3}^{1}\right)\right]^{c}=\operatorname{span}\left(x^{3}, x^{2} \epsilon, x \epsilon^{2}, \epsilon^{3}\right)$, we have

$$
\begin{aligned}
\frac{1}{3 !} g_{3}^{1}(x, 0, \epsilon)= & \operatorname{Proj}_{\left[\operatorname{Im}\left(M_{3}^{1}\right)\right]^{c}} \frac{1}{3 !} \widetilde{f}_{3}^{1}(x, 0, \epsilon) \\
= & \frac{k \tau \sigma\left(c_{2}-b\right)}{\eta} \epsilon^{2} x-k \tau \eta(\eta+\omega) \epsilon x^{2} \\
& -\frac{k \tau \eta^{5}}{\sigma^{2}}(r-1)(p-1)(1-\phi)(\omega+p \eta)^{2} x^{3},
\end{aligned}
$$

which, together with (53), implies that when $k_{1}=0$, the normal form on the invariant local center manifold $y=0$ is given by

$$
\begin{aligned}
\dot{x}= & \left(-k \sigma \tau+\frac{k \tau \sigma\left(c_{2}-b\right)}{\eta} \epsilon\right) \epsilon x-k \tau \eta(\eta+\omega) \epsilon x^{2} \\
& -\frac{k \tau \eta^{5}}{\sigma^{2}}(r-1)(p-1)(1-\phi)(\omega+p \eta)^{2} x^{3}+\text { h.o.t. }
\end{aligned}
$$

By the center manifold theorem and the bifurcation theorem, see Carr [34], Guckenheimer and Holmes [35], Hale and Lunel [31], Wiggins [30], and the references cited therein, the dynamics of the delayed differential (9) is topologically equivalent to that of (63) at the sufficiently small neighborhood of $\epsilon=0$. Therefore, by the normal forms on the center manifold equations (9) and (63), the following results can be obtained immediately.

Theorem 8. Assume that $R_{0}=1$; that is, $\epsilon=0$.

(1) If $k_{1} \neq 0$, the zero solution of (9) is unstable.

(2) If $k_{1}=0$, the zero solution of (9) is stable due to $\left(k \tau \eta^{5} / \sigma^{2}\right)(r-1)(p-1)(1-\phi)(\omega+p \eta)^{2}>0$.

Theorem 9. Assume that $\beta=\beta_{0}+\epsilon$ and $|\epsilon|$ is a sufficiently small positive number.

(1) If $k_{1} \neq 0$, (9) undergoes a transcritical bifurcation at the zero solution.

(2) If $k_{1}=0$, (9) undergoes a generic pitchfork bifurcation at the zero solution.

\section{Sensitive Analysis}

Sensitivity indices allow us to measure the relative change in a variable when a parameter changes. The normalized forward sensitivity index of a variable to a parameter is the ratio of the relative change in the variable to the relative change in the parameter. When the variable is a differentiable function of the parameter, the sensitivity index may be alternatively defined using partial derivatives. Here, one adopts the following definition as described by Chitnis et al. [36].

Definition 10. The normalized forward sensitivity index of a variable, $u$, that depends differentiably on a parameter, $p$, is defined as $\gamma_{p}^{u}=(\partial u / \partial p) \times(p / u)$.

Generally speaking, initial disease transmission is directly related to the threshold parameter $R_{0}$, and the disease prevalence is directly related to the positive equilibrium $E^{*}$, specifically to the magnitude of $I^{*}$. In the following, we calculate the sensitivity indexes of $R_{0}$ and the positive equilibrium $E^{*}$ to the parameters in system (6). The values of parameters used in this section are chosen from the literatures Gibson and Aritua [3], Holt and Chancellor [37], and van den Bosch et al. [10], as shown in Table 1.

Table 2 shows the sensitivity indices of $R_{0}$ with respect to each of the seven parameters in system (6). The indices with positive signs increase the value of $R_{0}$ when they are increased and those having negative signs decrease the value of $R_{0}$ when they are increased. It can be seen from Table 2 that $\gamma_{\phi}^{R_{0}} \epsilon$ $[-0.338963,0], \gamma_{r}^{R_{0}} \in[-0.022013,0], \gamma_{p}^{R_{0}} \in[-0.033138,0]$, $\gamma_{\eta}^{R_{0}} \in[-1.860169,-1.353863]$, and $\gamma_{\beta}^{R_{0}}=\gamma_{\sigma}^{R_{0}} \in[0.746847,1]$ as $\phi$ is varying in $[0,1]$. The most sensitive parameters to $R_{0}$ are the transmission rate $\beta$ and the planting rate $\sigma$, which means that increasing (or decreasing) $\beta$ and $\sigma$ by $10 \%$ increases (or decreases) $R_{0}$ by more than $7.46 \%$. Obviously, to maintain the reproduction of a plant, it is not practical to reduce $R_{0}$ by decreasing the planting rate $\sigma$. We also observe that $R_{0}$ is most sensitive to the harvest rate $\eta$ in an inversely proportional way. That is to say $R_{0}$ is a decreasing function of $\eta$, where increasing $\eta$ will decrease $R_{0}$ and decreasing $\eta$ will increase $R_{0}$. Furthermore $R_{0}$ is a decreasing function of $\phi$, $r, p$, and $\omega$. These results suggest that intervention strategies should be targeted to transmission rate $\beta$, harvest time $1 / \eta$, fraction planted from in vitro propagated, virus free, material 
TABLE 1: Description of variables and parameters in model (6).

\begin{tabular}{lccc}
\hline Parameter & Description & Estimated values & Reference \\
\hline$\phi$ & Fraction planted from virus free material & - & - \\
$r$ & Ratio of reversion & 0.08 & van den Bosch et al. [10] \\
$p$ & Probability of detecting an infected cutting & $(0,0.2316)$ & van den Bosch et al. [10] \\
$\eta$ & Harvest rate & $2 \times 10^{-3}$ & Holt and Chancellor [37] \\
$\beta$ & Transmission rate & $6.4 \times 10^{-3}$ & van den Bosch et al. [10] \\
$\sigma$ & Planting rate & $1.5 \times 10^{-3}$ & Gibson and Aritua [3] \\
$\omega$ & Roguing rate & $3.3 \times 10^{-4}$ & van den Bosch et al. [10] \\
\hline
\end{tabular}

TABLE 2: Sensitivity indices of $R_{0}$ to all parameters.

\begin{tabular}{lccccccc}
\hline Parameter $j$ & $\phi$ & $r$ & $p$ & $\eta$ & $\beta$ & $\sigma$ \\
\hline$\gamma_{j}^{R_{0}}$ & $\frac{3 \times 10^{-6} \phi}{l_{1}(\phi)}$ & $\frac{3 \times 10^{-7}(\phi-1)}{l_{1}(\phi)}$ & $\frac{4 \times 10^{-7}(\phi-1)}{l_{1}(\phi)}$ & $-\frac{l_{2}(\phi)}{l_{1}(\phi)}$ & $\frac{1 \times 10^{-5}}{l_{1}(\phi)}$ & $\frac{1 \times 10^{-5}}{l_{1}(\phi)}$ & -0.139831 \\
\hline
\end{tabular}

where $l_{1}(\phi)=1.3 \times 10^{-5}-3 \times 10^{-6} \phi$ and $l_{2}(\phi)=1.7 \times 10^{-5}+5 \times 10^{-7} \phi$.

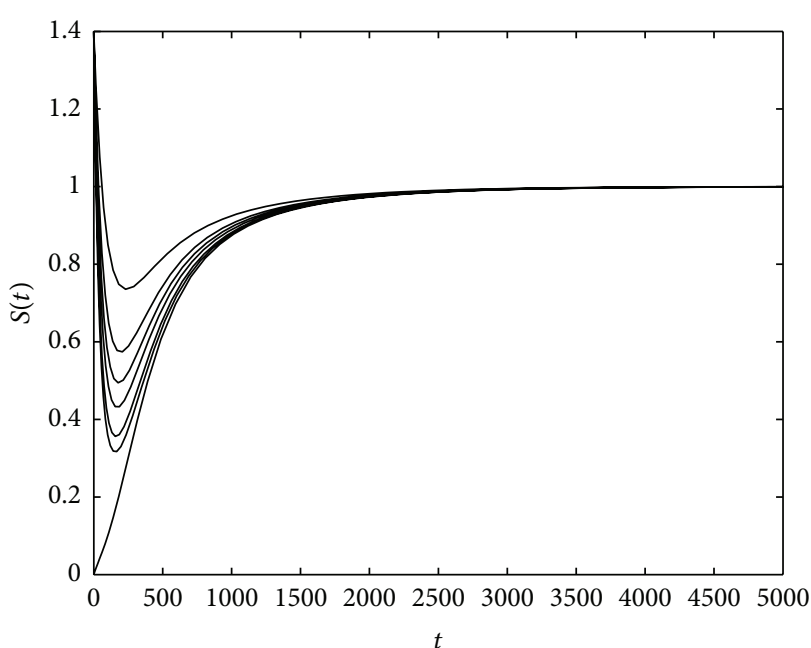

(a)

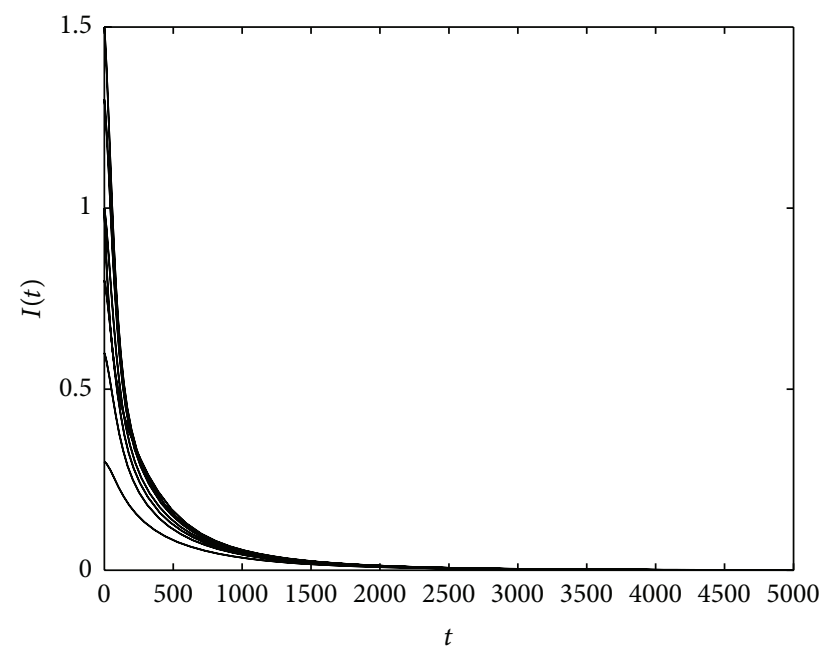

(b)

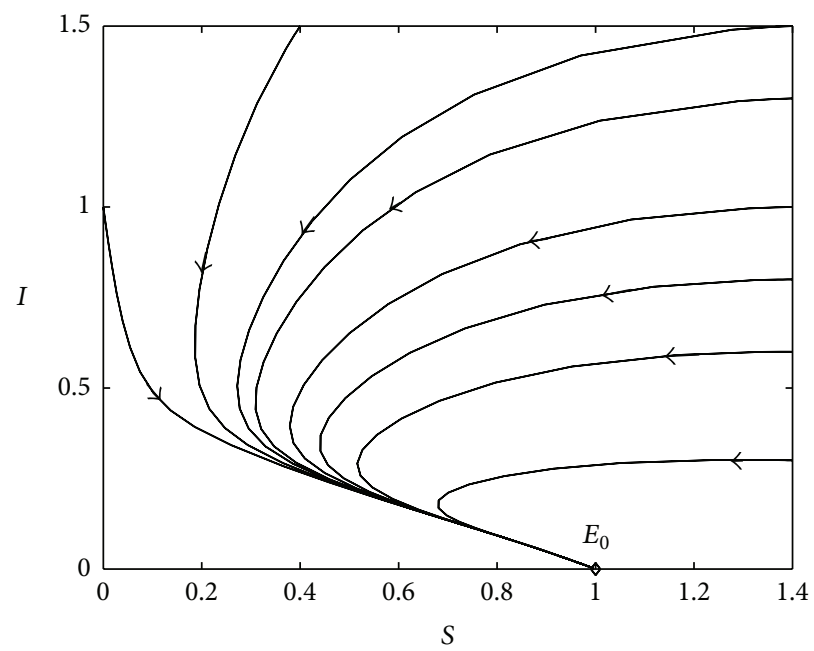

(c)

Figure 1: $\tau=0, R_{0}<1$. The solutions of system (6) approach to the disease-free equilibrium $E_{0}=(1,0)$. 


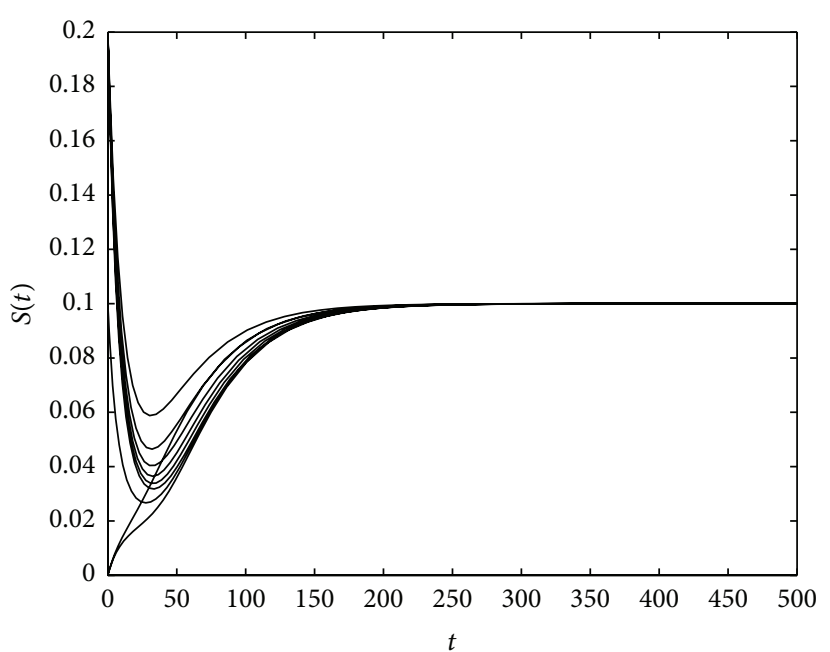

(a)

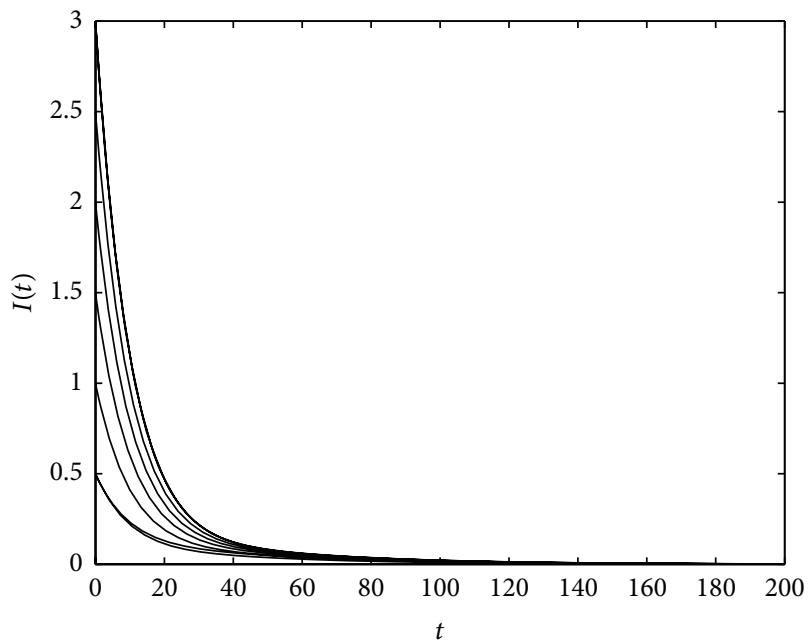

(b)

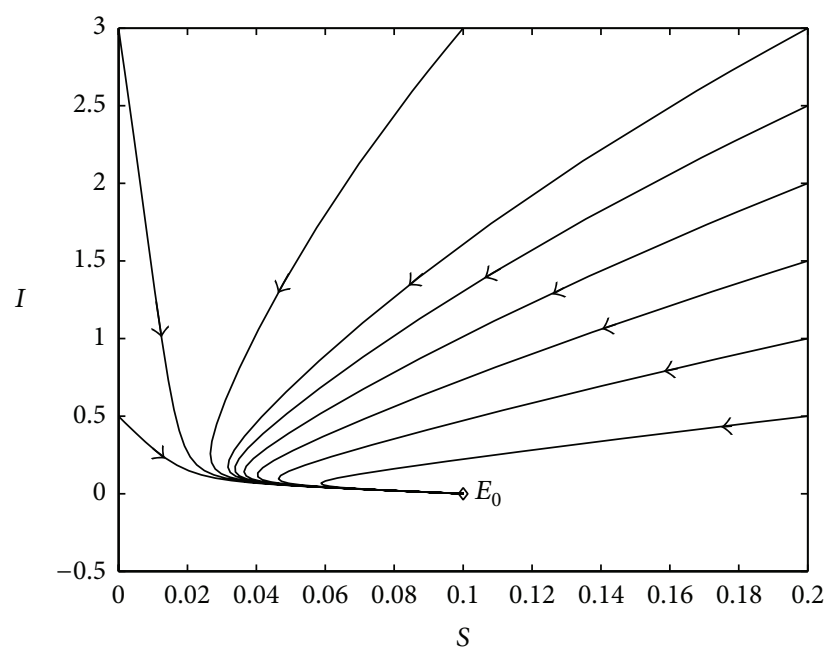

(c)

FIgURE 2: $\tau>0, R_{0}<1$. The solutions of system (6) tends to the disease-free equilibrium $E_{0}=(0.1,0)$.

TABLE 3: Sensitivity indices of $E^{*}$ with $\phi=0.2$.

\begin{tabular}{lccc}
\hline Parameter & $S^{*}$ & $I^{*}$ & $N^{*}$ \\
\hline$\phi$ & +0.663446 & -0.110946 & +0.552500 \\
$r$ & +0.230764 & -0.038590 & +0.192174 \\
$p$ & +0.107352 & -0.010476 & +0.096876 \\
$\mathrm{~W} \eta$ & +1.006333 & -1.195680 & -0.189352 \\
$\beta$ & -1.114529 & +0.163789 & -0.928150 \\
$\sigma$ & -0.114529 & +1.186579 & +1.072050 \\
$\omega$ & +0.222730 & -0.177077 & +0.045653 \\
\hline
\end{tabular}

$\phi$, the ratio of reversion $r$, the probability of detecting an infected cutting $p$, and the roguing rate $\omega$.

As the analytical expressions for the sensitivity indices of the positive equilibrium $E^{*}$ do not possess good structures, we therefore evaluate the sensitivity indices at the parameter values in Table 1, and then show the sensitivity indices of the state variables for low and high planting rates of virus free material (e.g., $\phi=0.2,0.8$ ) in Tables 3 and 4 , respectively.

It can be seen from Tables 3 and 4 that the most sensitive parameter to the population size $N^{*}$ is the planting rate $\sigma$ for both the low and high proportions of virus free material, followed in an inverse way by the transmission rate $\beta$ and the ratio of reversion $r$. The most sensitive parameter to the infected plant $I^{*}$ is the harvest rate $\eta$ in an inverse way, followed by the planting rate $\sigma$. The most sensitive parameter to the susceptible plant $S^{*}$ is the transmission rate $\beta$ in an inverse way, followed by the harvest rate $\eta$.

\section{Number Simulations}

Numerical simulation is an effective method to investigate the properties of system (6). In this section, we present numerical simulations to support the theoretical analysis given in Sections 2 and 3. 


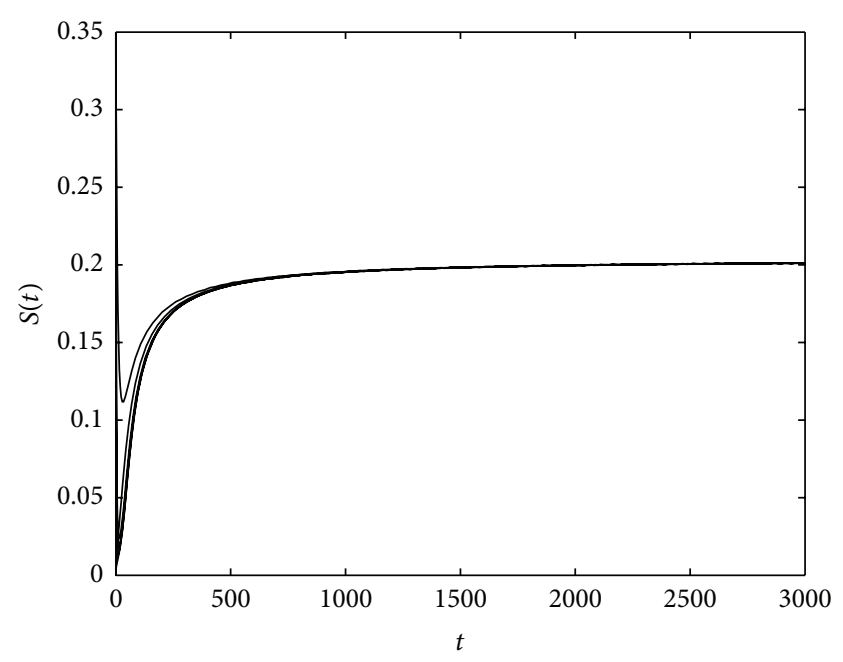

(a)

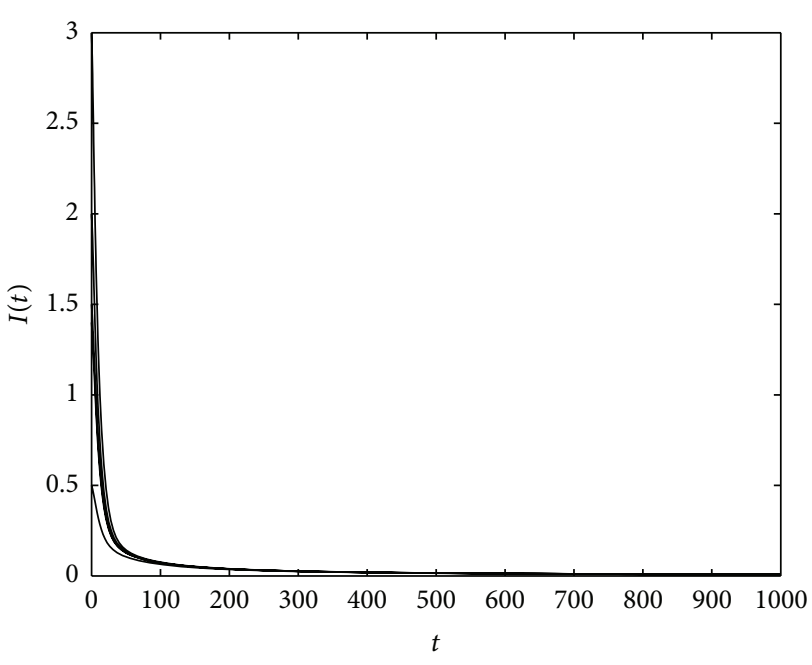

(b)

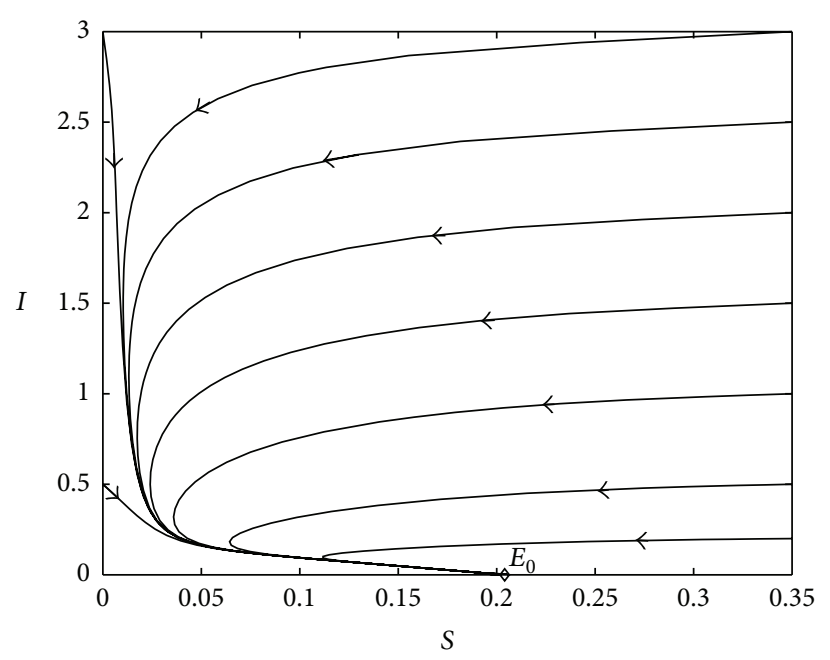

(c)

FIgURE 3: $\tau>0, R_{0}=1$. The solutions of system (6) tends to the disease-free equilibrium $E_{0}=(0.20405,0)$.

TABLE 4: Sensitivity indices of $E^{*}$ with $\phi=0.8$.

\begin{tabular}{lccc}
\hline Parameter & $S^{*}$ & $I^{*}$ & $N^{*}$ \\
\hline$\phi$ & +0.797581 & -0.543699 & +0.253882 \\
$r$ & +0.017339 & -0.011820 & +0.005519 \\
$p$ & +0.041648 & -0.008413 & +0.033235 \\
$\eta$ & +0.888967 & -2.147856 & -1.258887 \\
$\beta$ & -1.022566 & +0.697064 & -0.325502 \\
$\sigma$ & -0.022566 & +1.697066 & +1.674500 \\
$\omega$ & +0.156165 & -0.246287 & -0.090121 \\
\hline
\end{tabular}

Firstly, let $\tau=0, \beta=0.01, \phi=0.1, p=0.01, r=0.01$, $\omega=0.01, \sigma=0.01$, and $\eta=0.01$. Then, it is easy to obtain that $R_{0} \approx 0.94<1$. From the second case of Theorem 2 , the disease-free equilibrium is asymptotically stable if $R_{0}<1$ and $\tau=0$. Figure 1 exhibits that all of the solutions approach the disease-free equilibrium $E_{0}$.
Secondly, let $\tau=0.5, \phi=0.1, p=0.1, r=0.1$, $\eta=0.1, \omega=0.001, \sigma=0.01$, and $\beta=0.01$. Then, it is easy to obtain that $R_{0} \approx 0.73<1$. From Theorem 4 , the disease-free equilibrium is asymptotically stable if $R_{0}<1$ and $\tau>0$. Figure 2 exhibits that all of the solutions approach the disease-free equilibrium $E_{0}$.

Finally, let $\tau=0.5, \phi=0.1, p=0.1, r=0.1, \eta=0.1, \omega=$ $0.01, \sigma \approx 0.0204$, and $\beta \approx 0.1818$. Then, we have $R_{0}=1$ and $k_{1}=0$. From the second case of Theorem 9 , the disease-free equilibrium is stable if $R_{0}=1$ and $k_{1}=0$. Figure 3 exhibits that all of the solutions approach the disease-free equilibrium $E_{0}$.

\section{Conclusions}

It is still an interesting topic to determine how the time delay affects overall disease progression and, mathematically, how the delay affects the dynamics of systems. In this paper, a delayed disease model with continuous cultural control 
strategy is formulated. By analyzing the transendental characteristic equation, we derive some conditions on the stability of the disease-free equilibrium. The disease-free equilibrium is stable, if $R_{0}<1$, and unstable if $R_{0}>1,0 \leq \tau<\tau_{0}$. Then, by virtue of the methods in Guckenheimer and Holmes [35] and Wiggins [30], the stability of the nonhyperbolic disease-free equilibrium is discussed if $R_{0}=1$ and $\tau=$ 0 . Furthermore, if $R_{0}=1, \tau>0$ and $\neq 1 / q_{1}$, zero is a simple root of the characteristic equation (20), and the delayed model (6) exhibits rich dynamics. Employing the center manifold reduction and normal form theory due to Faria and Magalhaes [32, 33], we compute the normal form of system (6) associated with zero eigenvalue. By analyzing the normal form, we get that the disease-free equilibrium is unstable if $k_{1} \neq 0$ and stable if $k_{1}=0$. From the view of bifurcation, system (6) undergoes transcritical bifurcation if $k_{1} \neq 0$ and generic pitchfork bifurcation if $k_{1}=0$ at the origin with small $\epsilon$.

Then, the sensitivity analysis of $R_{0}$ and the positive equilibrium $E^{*}$ is carried out in order to determine the relative importance of different factors responsible for disease transmission. The results show that the most sensitive parameters to $R_{0}$ are the transmission rate $\beta$ and the planting rate $\sigma$, the most sensitive parameter to the state variable $I^{*}$ is the harvest rate $\eta$ followed by the planting rate $\sigma$, the most sensitive parameter to the state variable $S^{*}$ is the transmission rate $\beta$ followed by the harvest rate $\eta$, and the most sensitive parameter to the population size $N^{*}$ is the planting rate $\sigma$ for both the low and high proportions of virus free material $\phi$, followed by the transmission rate $\beta$ and the ratio of reversion $r$.

At last, numeric simulations are used to verify the qualitative results obtained in the present paper.

\section{Conflict of Interests}

The authors declare that there is no conflict of interests regarding the publication of this paper.

\section{Acknowledgments}

This work is supported by the Natural Science Foundation of China (nos. 11201277, 11271125, 11271236, and 10971064), the Scientific Research Plan Projects of Shaanxi Education Department (nos. 12JK0851 and 2013JK0611), Project for Higher Education Research of Xian University of Sciences and Technology (no. GJY2013-ZD-2), and Fluid Mechanicsthe Elaborate Course of Xian University of Science and University (2013) and the Key Laboratory of Simulation and Control for Population Ecology (Xinyang Normal University), Xinyang 464000, China (no. 201004).

\section{References}

[1] E. P. Rybicki and G. Pietersen, "Plant virus disease problems in the developing world," Advances in Virus Research, vol. 53, pp. 127-175, 1999.

[2] G. Dahal, J. A. Hughes, and B. E. L. Lockhart, "Status of banana streak disease in Africa: problems and future research needs,"
Integrated Pest Management Reviews, vol. 3, no. 2, pp. 85-97, 1998.

[3] R. W. Gibson and V. Aritua, "The perspective of sweet potato chlorotie stunt virus in sweet potato production in Africa, a review," African Crop Science Journal, vol. 10, no. 4, pp. 281-310, 2002.

[4] J. M. Thresh and R. J. Cooter, "Strategies for controlling cassava mosaic virus disease in Africa," Plant Pathology, vol. 54, no. 5, pp. 587-614, 2005.

[5] R. W. Gibson, J. P. Legg, and G. W. Otim-Nape, "Unusually severe symptoms are a characteristic of the current epidemic of mosaic virus disease of cassava in Uganda," Annals of Applied Biology, vol. 128, no. 3, pp. 479-490, 1996.

[6] J. J. Cho, D. M. Custer, S. H. Brommonschenkel et al., "Conventional breeding: host-plant resistance and the use of molecular markers to develop resistance to tomato spotted wilt virus in vegetables," Acta Horticulturae, vol. 431, pp. 367-378, 1996.

[7] R. A. C. Jones, "Determining "threshold" levels for seed-borne virus infection in seed stocks," Virus Research, vol. 71, no. 1-2, pp. 171-183, 2000.

[8] R. A. C. Jones, "Using epidemiological information to develop effective integrated virus disease management strategies," Virus Research, vol. 100, no. 1, pp. 5-30, 2004.

[9] R. A. C. Jones, "Developing integrated disease management strategies against non-persistently aphid-borne viruses: a model programme," Integrated Pest Management Reviews, vol. 6, no. 1, pp. 15-46, 2001.

[10] F. van den Bosch, M. J. Jeger, and C. A. Gilligan, "Disease control and its selection for damaging plant virus strains in vegetatively propagated staple food crops; a theoretical assessment," Proceedings of the Royal Society B, vol. 274, no. 1606, pp. 11-18, 2007.

[11] C. M. S. Chan Man Suen and M. J. Jeger, "An analytical model of plant virus disease dynamics with roguing and replanting," Journal of Applied Ecology, vol. 31, no. 3, pp. 413-427, 1994.

[12] S. Fishman, R. Marcus, H. Talpaz et al., "Epidemiological and economic models for spread and control of citrus tristeza virus disease," Phytoparasitica, vol. 11, pp. 39-49, 1983.

[13] F. Brauer and P. van den Driessche, "Models for transmission of disease with immigration of infectives," Mathematical Biosciences, vol. 171, no. 2, pp. 143-154, 2001.

[14] S. Y. Tang, Y. N. Xiao, and R. A. Cheke, "Dynamical analysis of plant disease models with cultural control strategies and economic thresholds," Mathematics and Computers in Simulation, vol. 80, no. 5, pp. 894-921, 2010.

[15] J. B. Sinclair, "Latent infection of soybean plants and seeds by fungi," Plant Disease, vol. 75, pp. 220-224, 1991.

[16] T. Ames, N. E. J. M. Smit, A. R. Braun, J. N. O'Sullivan, and L. G. Skoglund, Sweetpotato: Major Pests, Diseases, and Nutritional Disorders, International Potato Center, Lima, Peru, 2004.

[17] A. K. Miles, S. L. Willingham, and A. W. Cooke, "Field evaluation of strobilurins and a plant activator for the control of citrus black spot," Australasian Plant Pathology, vol. 33, no. 3, pp. 371-378, 2004.

[18] S. Chakraborty, "How will plant diseases impact on pasture production under climate change: a case study of stylosanthes anthracnose," in Proceedings of the 18th International Grasslands Congress, pp. 9-5-9-6, 1997.

[19] G. S. Karaoglanidis, Y. Luo, and T. J. Michailides, "Competitive ability and fitness of Alternaria alternata isolates resistant to QoI fungicides," Plant Disease, vol. 95, no. 2, pp. 178-182, 2011. 
[20] J. E. van der Plank, Plant Diseases: Epidemics and Control, Academic Press, London, UK, 1963.

[21] L. V. Madden, "Botanical epidemiology: some key advances and its continuing role in disease management," European Journal of Plant Pathology, vol. 115, no. 1, pp. 3-23, 2006.

[22] J. D. Murray, Mathematical Biology: I. An Introduction, Interdisciplinary Applied Mathematics, Springer, Berlin, Germany, 2002.

[23] P. S. Teng, "A comparison of simulation approaches to epidemic modeling," Annual Review of Phytopathology, vol. 23, pp. 351379, 1985.

[24] J. C. Zadoks, "Systems analysis and the dynamics of epidemics," Phytopathology, vol. 61, pp. 600-610, 1971.

[25] M. J. Jeger, "Asymptotic behavior and threshold criteria in model plant disease epidemics," Plant Pathology, vol. 35, no. 3, pp. 355-361, 1986.

[26] M. J. Jeger, "Relation between rate parameters and latent and infectious periods during a plant disease epidemic," Phytopathology, vol. 74, pp. 1148-1152, 1984.

[27] A. C. Kushalappa and A. Ludwig, "Calculation of apparent infection rate in plant diseases: development of a method to correct for host growth," Phytopathology, vol. 72, pp. 1373-1377, 1983.

[28] P. E. Waggoner, "Contributions of mathematical models to epidemiology," Annals of the New York Academy of Sciences, vol. 287, pp. 191-206, 1977.

[29] N. J. Cunniffe, R. O. J. H. Stutt, F. van den Bosch, and C. A. Gilligan, "Time-dependent infectivity and flexible latent and infectious periods in compartmental models of plant disease," Phytopathology, vol. 102, no. 4, pp. 365-380, 2012.

[30] S. Wiggins, Introduction to Applied Nonlinear Dynamical Systems and Chaos, Texts in Applied Mathematics, Springer, Berlin, Germany, 1990.

[31] J. K. Hale and S. M. V. Lunel, Introduction to FunctionalDifferential Equations, Applied Mathematical Sciences, Springer, New York, NY, USA, 1993.

[32] T. Faria and L. T. Magalhaes, "Normal forms for retarded functional-differential equations and applications to BogdanovTakens singularity," Journal of Differential Equations, vol. 122, no. 2, pp. 201-224, 1995.

[33] T. Faria and L. T. Magalhaes, "Normal forms for retarded functional-differential equations with parameters and applications to Hopf bifurcation," Journal of Differential Equations, vol. 122, no. 2, pp. 181-200, 1995.

[34] J. Carr, Applications of Centre Manifold Theory, Applied Mathematical Sciences, Springer, New York, NY, USA, 1981.

[35] J. Guckenheimer and P. Holmes, Nonlinear oscillations, Dynamical Systems, and Bifurcations of Vector Fields, Applied Mathematical Sciences, Springer, New York, NY, USA, 1983.

[36] N. Chitnis, J. M. Hyman, and J. M. Cushing, "Determining important parameters in the spread of malaria through the sensitivity analysis of a mathematical model," Bulletin of Mathematical Biology, vol. 70, no. 5, pp. 1272-1296, 2008.

[37] J. Holt and T. C. B. Chancellor, "A model of plant virus disease epidemics in asynchronously-planted cropping systems," Plant Pathology, vol. 46, no. 4, pp. 490-501, 1997. 


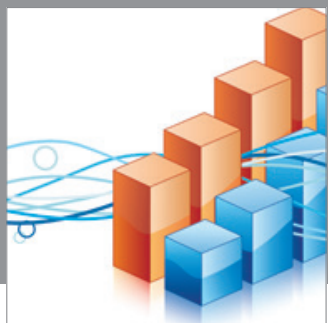

Advances in

Operations Research

mansans

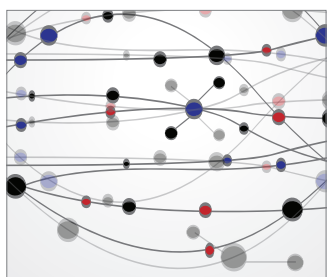

The Scientific World Journal
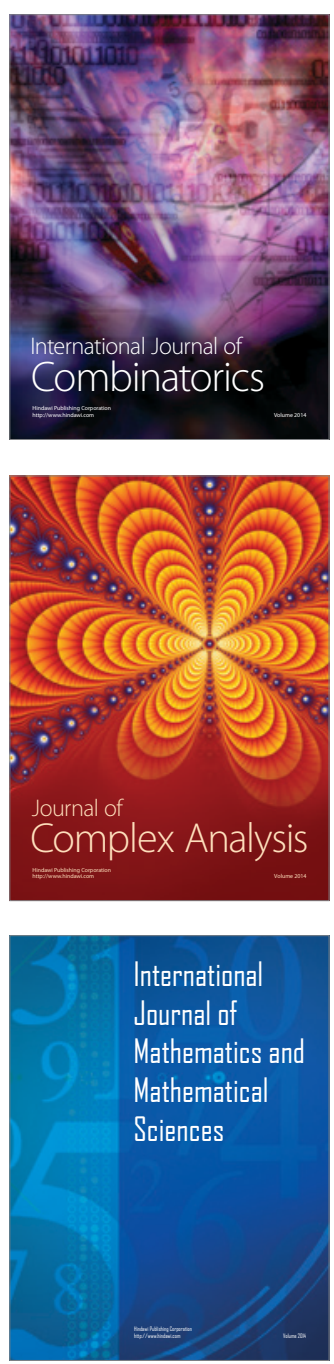
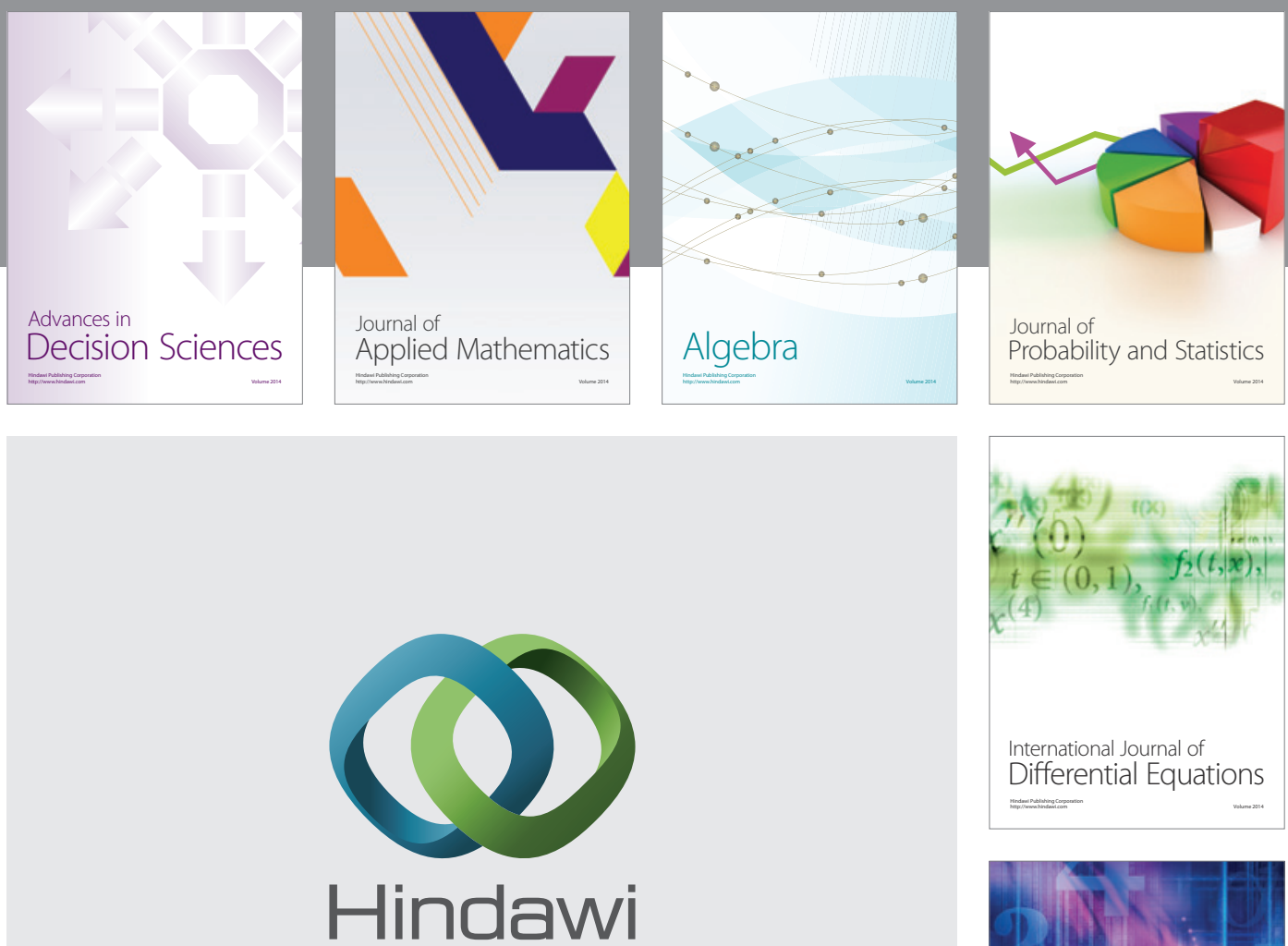

Submit your manuscripts at http://www.hindawi.com
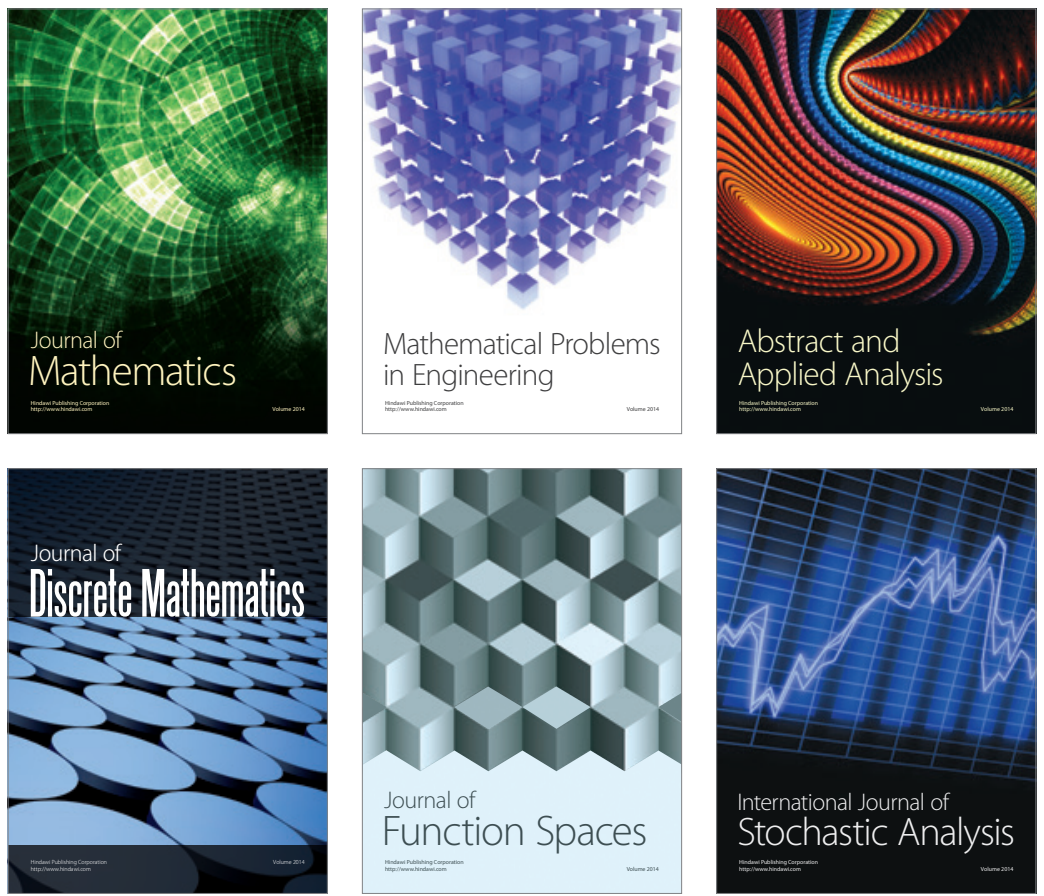

Journal of

Function Spaces

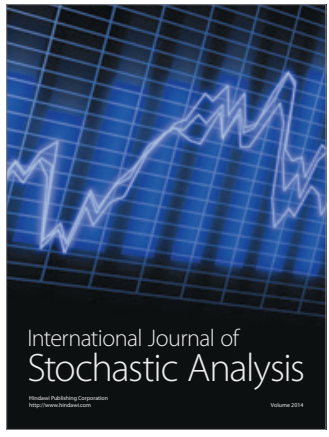

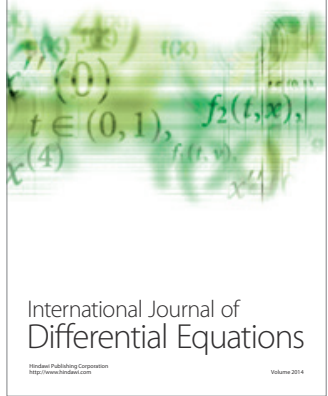
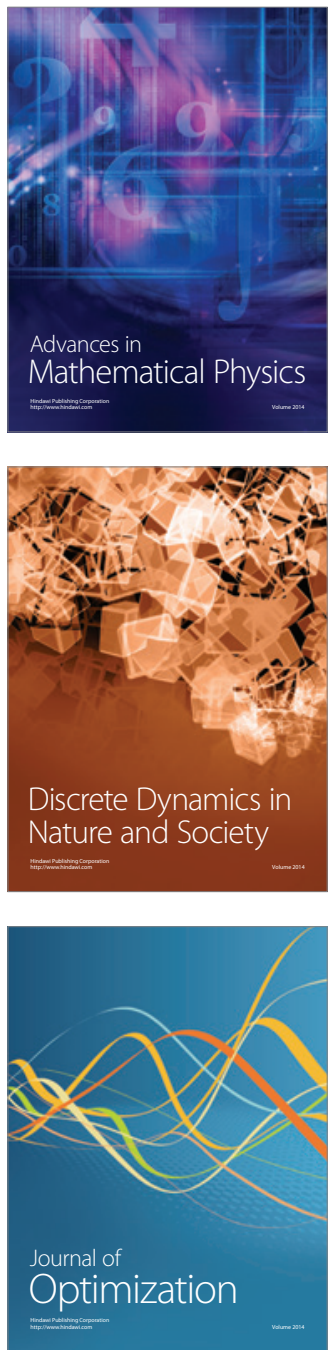